\title{
Connecting the dots: targeting gut microbiota in drug toxicity
}

\author{
Yusha Luo ${ }^{1}$ and Tingting Zhou ${ }^{1}$ \\ ${ }^{1}$ Second Military Medical University School of Pharmacy
}

June 8, 2020

\begin{abstract}
Gut microbiota has been demonstrated to have a vast influence on human health in recent decades and its role in initiating, aggravating, or ameliorating diseases is emerging. Therapeutic strategies have therefore increasingly developed by targeting gut microbial modulation. Recently, its contribution to heterogeneous toxicological responses is also gaining attention, especially in drug-induced toxicity. Oral drugs interact directly with gut microbiota within the gastrointestinal tract and a number of them elicit toxicity mediated by intestinal microbiota. Present studies focus more on the unidirectional influence of how xenobiotics disturb intestinal microbial composition and function and consequently induce altered homeostasis. However, interactions between gut microbiota and xenobiotics are bidirectional and the impact of gut microbiota on xenobiotics especially on drugs should not be neglected. Thus, in this review, we intend to focus on how gut microbiota modulates drug toxicity by highlighting gut microbiota, microbial enzyme, and metabolites to proffer references for seeking common countermeasures in coping with drug toxicity by targeting gut microbiota. Moreover, we give a hypothesis that drugs capable of inducing gut dysbiosis tend to more or less impact the gut-connected organs as evidenced by the drug-induced hepatic encephalopathy, indicating an underlying link among the gut, liver, brain, and other possible organs in drug-induced toxicity.
\end{abstract}

\begin{abstract}
Gut microbiota has been demonstrated to have a vast influence on human health in recent decades and its role in initiating, aggravating, or ameliorating diseases is emerging. Therapeutic strategies have therefore increasingly developed by targeting gut microbial modulation. Recently, its contribution to heterogeneous toxicological responses is also gaining attention, especially in drug-induced toxicity. Oral drugs interact directly with gut microbiota within the gastrointestinal tract and a number of them elicit toxicity mediated by intestinal microbiota. Present studies focus more on the unidirectional influence of how xenobiotics disturb intestinal microbial composition and function and consequently induce altered homeostasis. However, interactions between gut microbiota and xenobiotics are bidirectional and the impact of gut microbiota on xenobiotics especially on drugs should not be neglected. Thus, in this review, we intend to focus on how gut microbiota modulates drug toxicity by highlighting gut microbiota, microbial enzyme, and metabolites to proffer references for seeking common countermeasures in coping with drug toxicity by targeting gut microbiota. Moreover, we give a hypothesis that drugs capable of inducing gut dysbiosis tend to more or less impact the gut-connected organs as evidenced by the drug-induced hepatic encephalopathy, indicating an underlying link among the gut, liver, brain, and other possible organs in drug-induced toxicity.
\end{abstract}

Key words:gut-liver axis; gut-brain axis; gut microbiota; hepatotoxicity; gastrointestinal toxicity; neurotoxicity.

Abbreviations : 4-HPA, 4-hydroxyphenylacetic acid; 5-FU, 5-fluorouracil; AChE, acetylcholinesterase; AFB1, aflatoxins B1; APAP, acetaminophen; ASD, autism spectrum disorders; BBB, blood brain barrier; CYPs, cytochrome 450 enzymes; DOPA, 3,4-dihydroxyphenylacetic acid; FGF, fibroblast growth factor; FMT, fecal microbiota transplantation; FXR, farnesoid X receptor; GABA, $\gamma$-aminobutyric acid; GSH, glutathione; HE, hepatic encephalopathy; LPS, lipopolysaccharide; MMF, mycophenolate mofetil; MPA, mycophenolic 
acid; MPAG, mycophenolic acid glucuronide; MTX, methotrexate; NAFLD, non-alcoholic liver disease; NAPQI, N-acetyl-p-benzoquinone imine; Nrf-2, nuclear factor erythroid 2-related factor 2; PPD, 1-phenyl1,2-propanedione; SCFAs, short-chain fatty acids; SN-38, SN-38 glucuronide; TLR, toll-like receptor; UGTs, UDP-glucuronosyltransferases.

\section{INTRODUCTION}

The microbiota has gained enough attention wherever in liver, brain, and other diseases in recent decades. Indeed, it is comparable with the liver in weight and outnumbers human cells by magnitudes (Qin et al., 2010), indicating its great potential in regulating human health and diseases. Presented as an integral microorganism system, microbiota resides in almost every niche of the human body and is mainly colonized in the skin, airways, urogenital, eyes, and gastrointestinal tract. Among the habitats of microbiota, gut harbors the majority of it, comprised of bacteria, archaea, fungi, viruses, and diverse other microorganisms (Leung et al., 2016). The prokaryote is the most abundant constituent of intestinal microbial microorganisms which can be classified into 11 phyla, with Proteobacteria, Firmicutes, Actinobacteria, and Bacteroidetes comprising over $90 \%$ of the gut microbiome. Some of the phyla have determinant significance in homeostasis maintenance, for instance, the Firmicutes / Bacteroidetes ratio is bound up with obesity (Koliada et al., 2017). Specific gut microbiota may secrete particular enzymes such as $\alpha$-Rhamnosidase, $\beta$-Glucuronidase, and $\beta$-Glucosidase (Gong et al., 2020) and produces metabolites including lipids, short-chain fatty acids (SCFAs), bile acids, vitamins, and amino acids (Fiori et al., 2020), which also are extensively engaged in disease pathogenesis. Since gut microbiota has been perceived as an important modulator of human health, numerous strategies including the use of germ-free animals, antibiotics and fecal microbiota transplantation (FMT) have been developed by targeting it, pushing it as a burgeoning area in physiological and pathological researches. Unsurprisingly, intestinal microbiota has been discovered to be implicated in ailments of the brain (Dinan and Cryan, 2017; Quigley, 2017), liver (Tilg et al., 2016), kidney (Mahmoodpoor et al., 2017), gastrointestinal tract (Gorkiewicz and Moschen, 2018; Nishida et al., 2018), and other parts of the human body and therapies including pre-/pro-/synbiotics supplementation, antibiotics treatment, diet intervention, and FMT have been implemented both in animal experiments and human trails. The intertwining of the gut with other organs derives many terms that connecting both, including the gut-liver/brain/kidney axis, each of which contains distinctive pathways but also interosculates in some. In other words, the gut serves as a bridge that connects other organs with the gut-liver-brain axis and gut-liver-kidney axis already put forward as key modulators in hepatic encephalopathy (HE) (Mancini et al., 2018) and chronic kidney disease (Yang et al., 2018a) pathogenesis respectively.

A plethora of marketed drugs have been reported with severe toxicity that confines their clinical application and some of them are compelled to withdraw from the market for unbearable toxicity. Since most drugs are orally administered, which means a majority of them especially those with low bioavailability are subject to gut microbiota (Dey, 2019), it is now proved to play a pivotal role in drug metabolism (Li et al., 2016; Walsh et al., 2018), pharmacokinetics (Zhang et al., 2018), and efficacy (Klaassen and Cui, 2015). The emerging of gut microbiota also provides a new perspective into understanding drug toxicity and manipulation of gut microbiota has been demonstrated with palliative effect in drug toxicity including hepatotoxicity, neurotoxicity, and gastrointestinal toxicity. Nowadays, evidence-based medicine highlights pharmacogenetics in deciphering and tackling variations of drug responses in patients (Relling and Evans, 2015) and since microbiota expresses tremendously more gene than the human host, gut microbial contribution to drug metabolism and toxicity also should be emphasized (Zimmermann et al., 2019).

Herein, we intend to give a brief introduction to gut-liver/brain axis first and then focus on drug hepatotoxicity, gastrointestinal toxicity, and neurotoxicity mediated by gut microbiota for the sake of connecting gut, liver and brain highlighting gut microbial function in modulating drug toxicity. Because each drug exhibits different metabolic characteristics that are pertinent to toxicity, this review is structured by drugs. Then, we elaborate the link of gut-liver-brain axis in $\mathrm{HE}$ and give some examples of drugs and chemicals that induce HE via gut-liver-brain axis. Finally, we summarize common targets by which gut microbiota impact drug toxicity and give our perspectives on future research directions of it. 


\section{GUT-LIVER/BRAIN AXIS}

As the saying goes, always trust your gut - it knows what your head and liver have not yet figured out, we have been searching for pathogenesis and therapies of brain and liver from gut in recent decades. Indeed, intestinal epithelium, gut microbial composition, and gut-derived enzyme and metabolites are all instrumental factors in the occurrence and development of numerous liver and brain diseases. The reciprocal interactions are termed into gut-liver/brain axis respectively. Below, a brief introduction of gut-liver/brain axis is provided, emphasizing gut microbial function in liver/brain diseases and pathways and mediators amid.

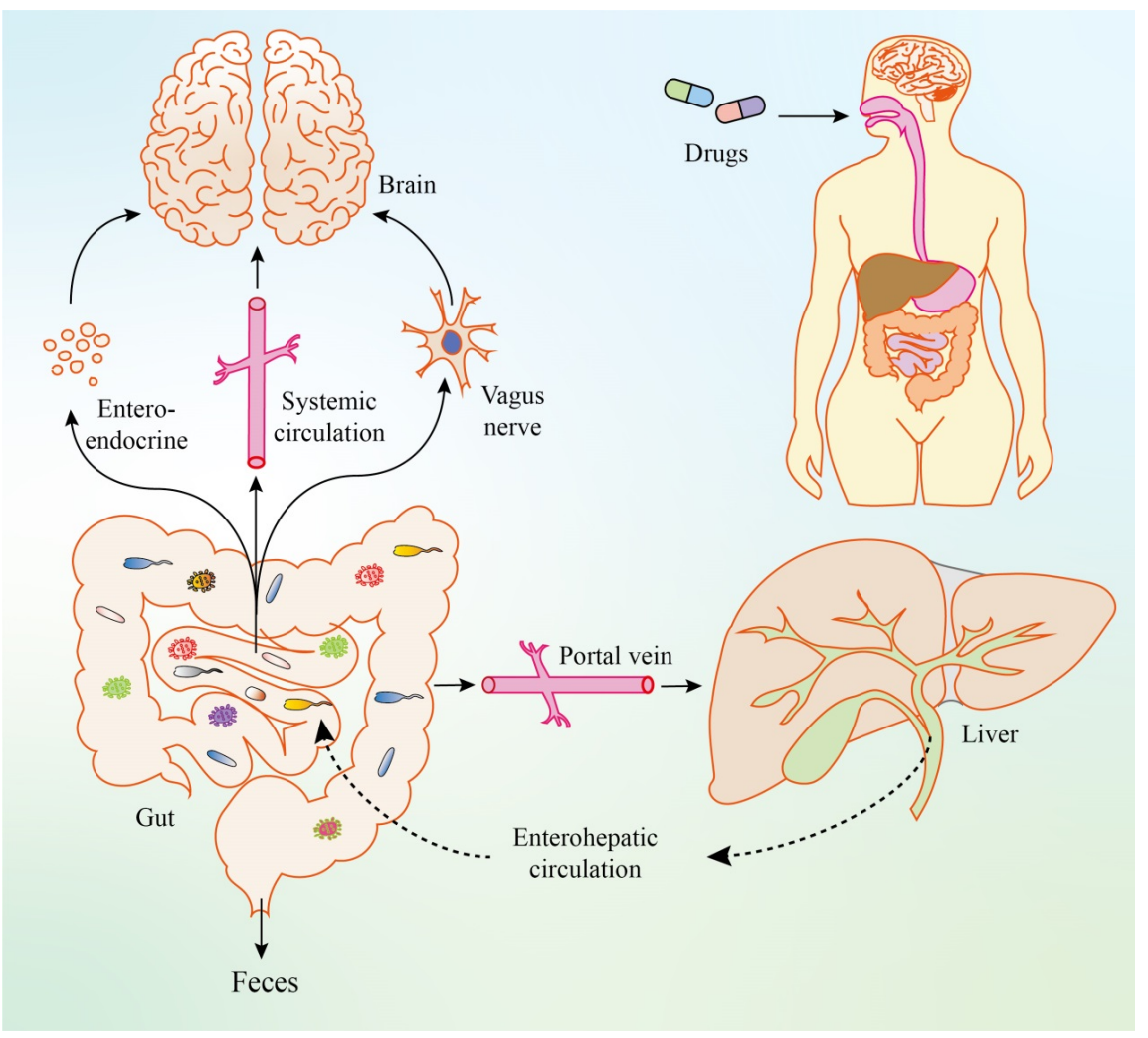

Fig.1 An overview of gut-liver-brain axis.

\subsection{Gut-liver axis}

The gut-liver axis is a hot topic in recent decades. There exists a delicate relationship between gut and liver for their anatomical and functional intimacy. After partially absorbed by the gastrointestinal tract, some xenobiotics such as oral drugs are subject to the liver for further metabolism through the portal vein and some of the metabolites accompanied with bile are recycled into the intestine via enterohepatic circulation (Roberts et al., 2002), indicating the intimate relationship between gut and liver (Fig.1). Hence, ever since gut microbiota was highlighted decades ago, scholars have been connecting gut microbiota with liver diseases which gains much progress in this field. Gut dysbiosis has been associated with the pathogenesis of a wide spectrum of liver diseases including alcoholic liver disease, non-alcoholic liver disease (NAFLD), hepatocellular carcinoma, autoimmune hepatitis, autoimmune cholangiopathies, acute liver injury, and liver fibrosis/cirrhosis (Hartmann et al., 2019). Notably, its role in initiating, perpetuating, and exacerbating NAFLD enables us to understand the etiology of the agnogenic and intricate liver disease and facilitates therapy of it (Leung et al., 2016). Mechanisms involved in gut microbiota related NAFLD include enterohepatic circulation of bile acids, increased intestinal permeability, systemic inflammation, altered immunity, and microbiota-derived metabolites, and so far as we know, manipulation of gut microbiota is fruitful in 
improving NAFLD with supplemental antibiotics and pre/pro/synbiotics, FMT, specific diet and exercise intervention having been demonstrated effective in NAFLD treatment, some of which are already in clinical trial phase (Wiest et al., 2017; Aron-Wisnewsky et al., 2020). Targets involved in gut microbiota mediated NAFLD locate in the gut lumen, gut mucosa, portal system, and liver/adipose tissue and gut dysbiosis modulates NAFLD negatively through microbiota-gut-liver axis by activating or inhibiting relevant receptors, cytokines or metabolites production therein (Leung et al., 2016) (Fig.2). Among the numerous metabolites engaged in the axis, bile acids are considered a common language of gut and liver with farnesoid X receptor (FXR) playing as a mediator. Primary bile acids are produced in the liver from cholesterol and are then subject to gut microbiota to form secondary bile acids. FXR, a nuclear receptor situated both in ileal enterocytes and hepatocytes, receives stimuli from liver secreted bile acids and activates expression of fibroblast growth factor (FGF) 19 in ileal enterocytes, which enters the liver via portal vein and activates FGF4/bklotho complex in hepatocytes and further inhibits cholesterol $7 \alpha$-hydroxylase (CYP7A1) expression, leading to suppression of bile acids synthesis (Wiest et al., 2017). Reversely, bile acids produced from liver to gut also influence gut microbial composition and function, constituting an important part in gut-liver bidirectional communication.

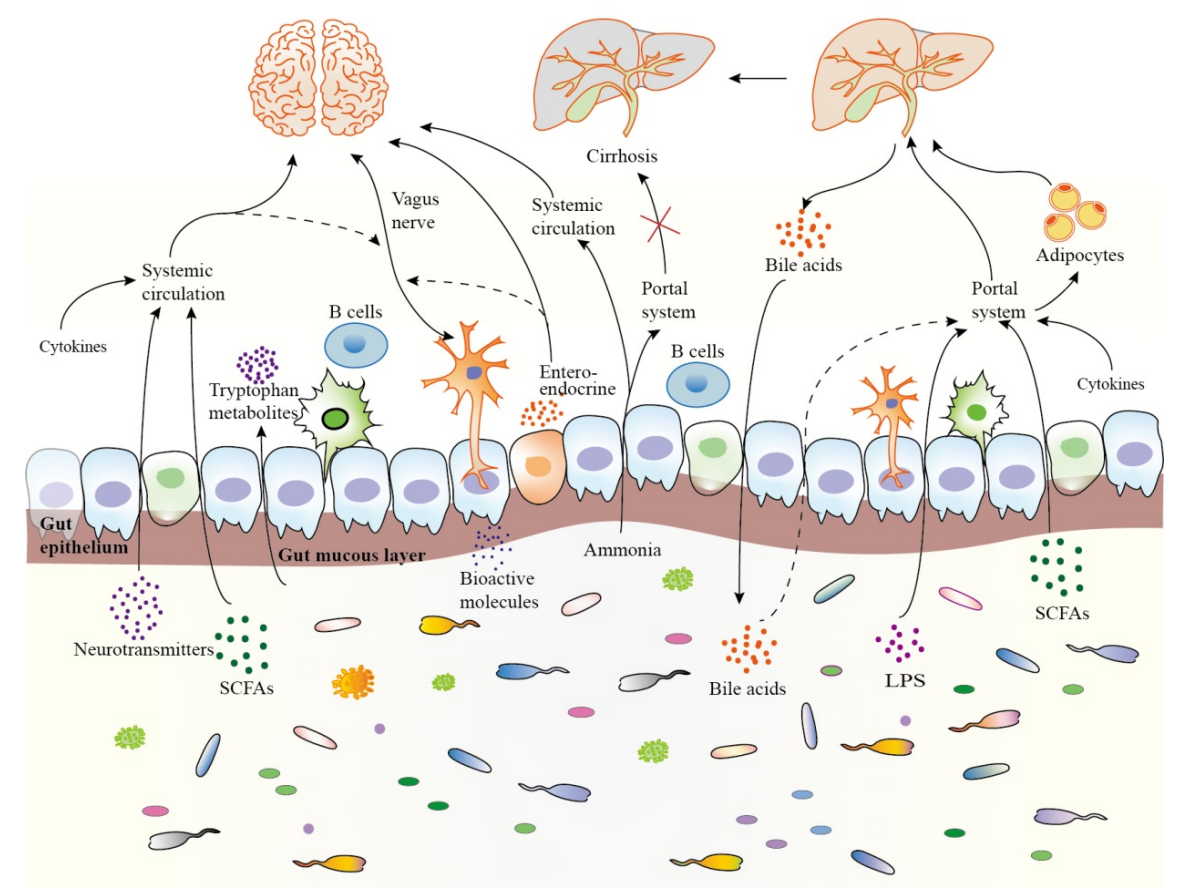

Fig.2 A schematic outline of gut-liver/brain interactions, focusing on pathways and representative metabolites. Abbreviations: LPS, lipopolysaccharide; NAFLD, non-alcohol fatty liver disease; SCFAs, short-chain fatty acids.

\subsection{Gut-brain axis}

Compared to the gut-liver axis, the gut-brain axis is a rather new concept that was initiated from a series of behavioral observations in germ-free and specific bacteria and antibiotics treated animals. It allows bidirectional communication between gut and brain, integrating nervous, endocrine, and immunological systems with gut and microbiota within (Fig.1). On the one hand, alterations in nervous, endocrine and immunological systems impact gut microbial composition and function, such as Helicobacter pylori infection (Budzyński and Kłopocka, 2014); on the other hand, modification of gut microbiota promotes or hampers mental health and cognitive function and has been presented as an attractive target for treating mental disorders such as 
Parkinson's disease (PD) (Fang, 2019), Alzheimer's disease (Khan et al., 2020), schizophrenia (Klein-Petersen et al., 2019), and autism (Aabed et al., 2019). Moreover, gut microbiota is involved in circadian rhythms of a wide spectrum of diseases including metabolic diseases, psychiatric disorders, and neurodegenerative diseases, along the gut-brain axis (Teichman et al., 2020). Pathways for reciprocal communication between gut microbiota and brain are situated in the gut lumen, gut epithelium, periphery, and brain (Fig.2), including hepatic and gallbladder metabolism, immune-modulatory responses, neuronal innervation, enteroendocrine, and microbial metabolite signaling (Cryan et al., 2019). Of note, hepatic and gallbladder metabolism is involved in the gut-brain axis with bile acids found to influence brain function directly (Liu et al., 2018), indicating an intimate relationship between liver and brain. Besides, gut-derived neurotransmitters such as $\gamma$-amino butyric acid (GABA), noradrenaline, and dopamine are especially significant gut microbial metabolites in bidirectional communication between the gut and brain. An intriguing fact is that both microbiota and brain development undergoes sensitive periods (early life, adolescence, and aging) during lifespan which may be implicitly intertwined since it is evidenced that antibiotic usage during perinatal stage may induce severe brain damage to the newborn and the aged are vulnerable to mental disorders with gut microbiota implicated. However, despite these flourishing discoveries of the gut-brain axis, many of the theories remain to be hypotheses, and much endeavor is needed to inject into fully elucidating mechanisms of how gut microbiota and brain communicate.

\section{GUT MICROBIOTA-MEDIATED DRUG HEPATOTOXICITY}

Drug-induced liver injury (DILI) is one of the most common liver injuries hitherto. Despite the findings and progress mentioned above, little is known about gut microbiota in DILI. The gut-liver axis, jointing the two major metabolic organs, deserves attention in mechanistic study of DILI but it is only recently that gut microbiota is found to be engaged in it. So in the following part of this review, we will discuss existing researches and findings concerning gut microbiota in DILI by concentrating on its role in the initiation, progression, and hindrance of drug-related hepatotoxicity (Fig.3).

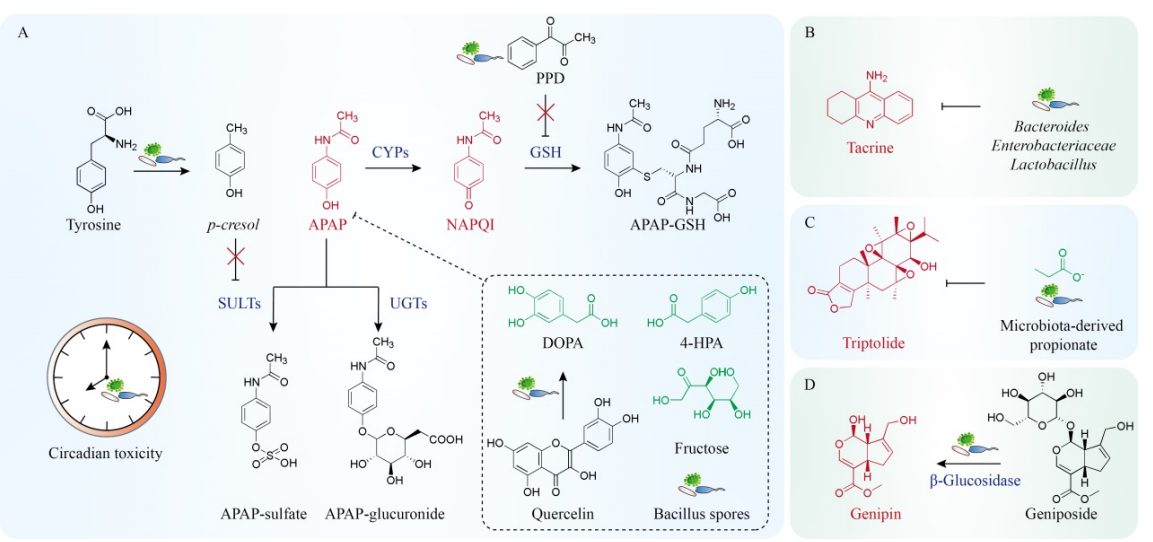

Fig.3 Drugs with hepatotoxicity modulated by gut microbiota: acetaminophen (A), tacrine (B), triptolide (C), and geniposide (D). Red structures signify hepatotoxic drugs or metabolites; green ones signify gutdervied metabolites that confers hepatoprotective effects against relevant drugs. A: PPD is a gut-derived metabolites that exacerbates APAP-induced hepatotoxicity by competing for GSH and so doesp-cresol which competes for sulfotransferase. DOPA, 4-HPA, and fructose are prebiotics that ameliorate APAP-induced hepatotoxicity. B: $\beta$-glucuronidase producing bacteria including Bacteroides, Enterobacteriaceae and Lactobacillus aggravate tacrine hepatotoxicity. C: Gut-derived propionate ameliorates triptolide-induced hepatotoxicity. D: Geniposide transforms into hepatotoxic genipin under the action of gut micorbiota. Abbreviations: 4-HPA, 4-hydroxyphenylacetic acid; APAP, acetaminophe; CYPs, cytochrome 450 proteins; DOPA, 3,4-dihydroxyphenylacetic acid; GSH, glutathione; NAPQI, N-acetyl-p-benzoquinone imine; SULTs, sulfotransferases; UGTs, UDP-glucuronosyltransferases. 


\subsection{Acetaminophen}

Acetaminophen (APAP) is a representative hepatotoxic drug accounting for most cases of DILI in the United States (Chao et al., 2018). When administered orally, it mainly undergoes two ways of metabolism: (a) sulfonated or glucuronidated by phase II enzymes and (b) first converted into the hepatotoxic N-acetyl-pbenzoquinone imine (NAPQI) by CYPs and then conjugated with glutathione (GSH) (Chang et al., 2020c). Both ways of metabolism function as detoxification and thus any factor that influences them could have an impact on APAP-related hepatotoxicity. A human experiment demonstrated that $p$-cresol, microbiotaderived metabolite from tyrosine, competed for sulfation with APAP, which might induce APAP accumulation and result in hepatotoxicity (Clayton et al., 2009). This experiment introduced gut microbiota into the understanding of APAP-related hepatotoxicity for the first time. Indeed, germ-free mice are less sensitive to APAP-induced hepatotoxicity compared with conventional mice (Possamai et al., 2015) and antibiotic treatment decreases urinary sulfonated APAP (Malfatti et al., 2020), suggesting that intestinal microbiota is implicated in APAP-related hepatotoxicity and metabolism. It has long been known that APAP-induced liver injury exhibits diurnal variation, which is more severe in the night than during the daytime (Kim and Lee, 1998). Despite the hepatic GSH level and drug metabolic activity mentioned as contributing factors of this phenomenon (Kim and Lee, 1998), the gut microbiota is thought to play a pivotal role therein. Evidence suggests that gut microbiota biogeography and metabolome undergo diurnal fluctuation, which consequently regulates rhythmic liver transcriptome and detoxification pattern as proved by APAP-induced circadian hepatotoxicity (Thaiss et al., 2016). The role of intestinal microbiota in rhythmic hepatotoxicity of APAP is further confirmed by another study and it also reported that 1-phenyl-1,2-propanedione (PPD), a gut microbial metabolite exacerbated APAP-induced liver injury by depleting hepatic GSH (Gong S, Lan T, Zeng L, Luo H, Yang X, Li N, Chen X, Liu Z, Li R, Win S, Liu S, Zhou H, Schnabl B, Jiang Y, Kaplowitz $\mathrm{N}, 2018$ ). A pharmacokinetic study demonstrated that treatment with probiotics, especiallyLactobacillus reuteri K8, diminished absorption of orally administered APAP (Kim et al., 2018), so it is speculated that pretreatment of moderate probiotics may ameliorate APAP-induced hepatotoxicity while maintaining efficacy simultaneously.

Based on the gut microbial mechanism of APAP-induced liver injury, some microbiota, diet-derived substances, and probiotics have been discovered to exert hepatoprotective effect against APAP by targeting the gut microbiota. 3,4-dihydroxyphenylacetic acid (DOPA), a microbial metabolite of quercetin, protects the liver from damage of APAP by activating nuclear factor erythroid 2-related factor 2 (Nrf-2) and enhancing phase II and antioxidant enzymes (Xue et al., 2016), so does 4-hydroxyphenylacetic acid (4-HPA), a major microbial metabolite of polyphenols (Zhao et al., 2018). As for food-derived hepatoprotective agents, a study found that supplemental fructose respited APAP-induced liver injury by altering gut microbial community, especially the genus Anaerostipes, which closely relates to hepatic levels of CYPs and GSH (Cho et al., 2017). Furthermore, probiotics like bacillus spores have been proven to protect rat from APAP-induced acute liver injury by altering proinflammatory cytokines levels (Neag et al., 2020). It seems gut microbial modulation does work in attenuating APAP-triggered liver injury, mainly by dietary modulation and probiotics intake, but it has to be verified in human trails for future application.

\subsection{Tacrine}

Tacrine was the first approved drug prescribed to treat Alzheimer's disease but was withdrawn from the market for its large pharmacokinetic variation (Jarrott, 2017) and consequent unpredictable hepatotoxicity (Bethesda (MD): National Institute of Diabetes and Digestive and Kidney Diseases, 2018). Although it is no longer used in clinical, underlying mechanisms involved in tacrine-induced liver injury is still being investigated (Park et al., 2015) and it is utilized to establish liver injury model in cells and animals (Stachlewitz et al., 1997; Choi et al., 2015). Previous researches mainly focused on the hepatic metabolism of tacrine and found that tacrine was extensively metabolized by CYP2A1 and one of the hydroxylated metabolites exerted hepatotoxicity (Madden et al., 1993). Fluvoxamine is a potent inhibitor of CYP2A1 and is thought to attenuate tacrine-induced hepatotoxicity by reducing the formation of toxic metabolites but needs further validation (Rasmussen et al., 1998). A recent comprehensive study combining pharmacokinetics, metabolomics, and 
metagenomics revealed that rats responded differently to tacrine with strong responders exhibiting higher tacrine exposure, greater deglucuronidation capabilities, and an abundance of $\beta$-glucuronidase producing bacteria including Bacteroides ,Enterobacteriaceae and Lactobacillus (Yip et al., 2018). Further validation indicated that coadministration of oral $\beta$-glucuronidase or antibiotics with tacrine increased or decreased susceptibility to tacrine-induced hepatotoxicity, indicating that gut microbiota especially the gut microbial $\beta$-glucuronidase plays a pivotal role in tacrine-related hepatotoxicity (Yip et al., 2018). It implicates the significance of gut microbiota in interindividually varied toxicity of drugs. While current researches attempt to decipher individual medication differences by genome but with limited achievements, the future focus can be shifted to gut microbiota.

\subsection{Triptolide}

Hepatotoxicity is one of the major factors that hamper the application and progress of herbal medicine nowadays. Triptolide is the major bioactive component of the herb Tripterygium wilfordii Hook $\mathrm{F}$ which exerts anti-inflammatory and anti-autoimmune functions but also confers hepatotoxicity. A study elucidated that triptolide exerted anti-inflammatory effect against ulcerative colitis with gut microbiota involved (Wu et al., 2020), indicating an interactive relationship between gut microbiota and triptolide. Molecular mechanisms of triptolide-induced hepatotoxicity have been comprehensively delineated, encompassing membrane damage, mitochondrial disruption, metabolism dysfunction, endoplasmic reticulum stress, oxidative stress, apoptosis, and autophagy (Xi et al., 2017), but the role of gut microbiota plays in it is put forward only recently. Researchers found that pretreatment of antibiotics exacerbated liver injury induced by triptolide in mice while supplemental microbiota-derived propionate proffered liver protection against triptolide (Huang et al., 2020a). It reveals that gut microbiota protects the liver from triptolide, contrary to the case in tacrine. Microbial metabolites of the prototype drugs may be an important factor herein, which indicates that microbial metabolites of triptolide confer lower hepatotoxicity while those of tacrine higher. Indeed, gut microbiota plays a unique role in triptolide metabolism since gut microbiota and liver microsomes produce different metabolites of triptolide (Peng et al., 2020). It indicates that gut microbial metabolism constitutes a significant part in drug metabolism and under some circumstances it influences and even determines toxic outcomes of drugs. Besides, triptolide treatment induced bile acids accumulation in rats and it was associated with suppression of FXR and hepatic Sirtuin (Sirt1) expression, activation of which ameliorated triptolide-induced hepatotoxicity (Yang et al., 2017).

\subsection{Geniposide}

Geniposide is the major bioactive component of herb Gardeniae Fructus which exhibits a wide range of pharmacological functions including neuroprotective, hypoglycemic, hepatoprotective, anti-inflammatory, analgesic, cardio-protective, antioxidant, immune-regulatory, antithrombotic, and antitumoral effects (Zhou et al., 2019b). The herb has long been used as choleretic to treat jaundice in Chinese history and modern research also reveals that geniposide attenuates APAP-induced liver injury in mice (Yang et al., 2019). Nonetheless, it has been reported that geniposide elevated serum alanine aminotransferase (ALT) and aspartate aminotransferase (AST) levels in rats and the conversion of geniposide to genipin made a big difference therein (Yamano et al., 1990). Genipin, the aglycon of geniposide, is produced from geniposide by intestinal bacteria but not by the liver (Aburada and Kobashi, 1994) and displayed much higher toxicity than geniposide in HepG2 cells (Kang et al., 2012), indicating the important role of gut microbiota in geniposideinduced liver injury. Antibiotic treatment of rats enhanced in vivo exposure of geniposide by suppressing metabolic activities of gut microbiota (Jin et al., 2014) and it might reduce hepatotoxicity because of a decreased level of genipin. A further study demonstrating mechanism involved in geniposide-induced liver injury attributed it to bile acids synthesis alteration (Tian et al., 2017), but the role of intestinal microbiota plays in it remains to be investigated. A recent study also proved the significance of intestinal microbiota in geniposide-related hepatotoxicity and further demonstrated that genipin dialdehyde intermediate, which was capable of conjugating with amino acids spontaneously, was the origin of geniposide-induced hepatotoxicity (Li et al., 2019).

3.5 Substances other than drugs 
Besides drugs, the gut microbiota is also extensively engaged in other substances-induced hepatotoxicity which will be discussed here as well. Some of the researches are profound in mechanistic elucidations and can provide as references for mechanistic studies of drug-induced hepatotoxicity.

Aflatoxins B1 (AFB1), a kind of mycotoxin, causes liver fibrosis and hepatocellular carcinoma both in humans and livestock. AFB1 exposure caused a wide range of metabolic disturbances and particularly elevated gut microbial cometabolites including phenylacetylglycine and hippurate in rats (Zhang et al., 2011). Other metabolomics based studies also found that AFB1 disrupted several important metabolic pathways of gut microbiota, including synthesis of SCFAs, pyruvic acid-related pathways, amino acids, bile acids, and long-chain fatty acids metabolism (Zhou et al., 2018b, 2019a), suggesting that gut microbial metabolism disruption contributes to the pathogenesis of AFB1-induced diseases. Probiotics supplementation including Lactobacillus Species (Liew et al., 2019; Wacoo et al., 2020), Propionibacterium freudenreichii(El-Nezami et al., 2006), Bacillus subtilis , and Candida utilis (Chang et al., 2020b) exerted protective effects against AFB1 both in animal experiments and human trials. These studies illustrate that AFB1 perturbs gut microbiota which can be restored by probiotics and they also help degrade AFB1 and thus lower toxicity.

Some simple substances form another main category of environmental toxicants that exert hepatotoxicity. Long-term exposure of low-dose cadmium causes severe liver damage with gut microbiota implicated. It was found that cadmium induced gut dysbiosis, mainly decreasing Firmicutes and $\gamma$-proteobacteria levels, and increasing serum lipopolysaccharide (LPS) and consequently induced hepatic inflammation (Zhang et al., 2015). It also increased intestinal permeability and hence inflicted accumulation in the liver but could be partially restored by antibiotic treatment (Liu et al., 2020). Arsenic is another example here. Although it is structurally simple, it undergoes complex biotransformation and forms a series of products with different toxicity. In vitro incubation elucidated the unique metabolic role of gut microbiota and in vivo study illustrated that gut microbiota reduced arsenic load by promoting methylation and thus protected the host from hepatotoxicity of arsenic (Chi et al., 2019a). Similarly, another study also found that a stable gut microbiome was a key determinant of survival to arsenic exposure, and Faecalibacterium prausnitzii protected it (Coryell et al., 2018).

Other environmental toxicants that are found to elicit hepatotoxicity with gut microbiota engaged include polychlorinated biphenyl 126 (Chi et al., 2019b), 2,3,7,8-tetrachlorodibenzo- $p$-dioxin (Fader et al., 2017), titanium dioxide nanoparticles (Chen et al., 2019), etc. These studies share similarities in researching method: first, 16S rRNA sequencing analysis is utilized to demonstrate gut dysbiosis-induced by the toxicants and then omics analysis especially metabolomics is used to elucidate disturbed metabolites or metabolic pathways by focusing on gut microbial metabolites and further deduce possible mechanisms involved by analyzing results of both. It is a current mainstream in gut microbiota associated mechanistic studies particularly in researches of metabolic diseases (Zeng et al., 2020).

\section{GUT MICROBIOTA-MEDIATED DRUG GASTROINTESTINAL TOXICITY}

The gastrointestinal reaction is a commonly observed drug side effect especially for chemotherapeutical agents and is characterized by nausea, vomiting, diarrhea, and constipation (Ervin et al., 2020). Studies have revealed the pivotal role of gut microbiota in therapeutic and toxic outcomes of cancer therapy (Roy and Trinchieri, 2017; Gopalakrishnan et al., 2018; Gori et al., 2019) and gut microbiome can even be used as a tool to anticipate individual gastrointestinal toxicity (Wardill and Tissing, 2017). It is believed that reciprocal interactions between gut microbiota and innate immunity contributes to chemotherapyinduced gastrointestinal toxicity (Secombe et al., 2019) and modulation of gut microbiota by probiotics has been proven to attenuate gastrointestinal toxicity of cancer therapy in human trials (Ciorba et al., 2015). Chemotherapy-induced gastrointestinal side effects negatively influence nutritional status and life quality of patients but most importantly they intervene adherence to chemotherapy regiment, causing much difficulty in the process of cancer therapy and prognosis (Ervin et al., 2020) and therefore coping with this problem is of utmost importance in cancer therapy. Gut microbiota hitherto has been correlated with methotrexate, irinotecan, 5-fluorouracil, and mycophenolate mofetil-induced gastrointestinal toxicity (Fig.4). 


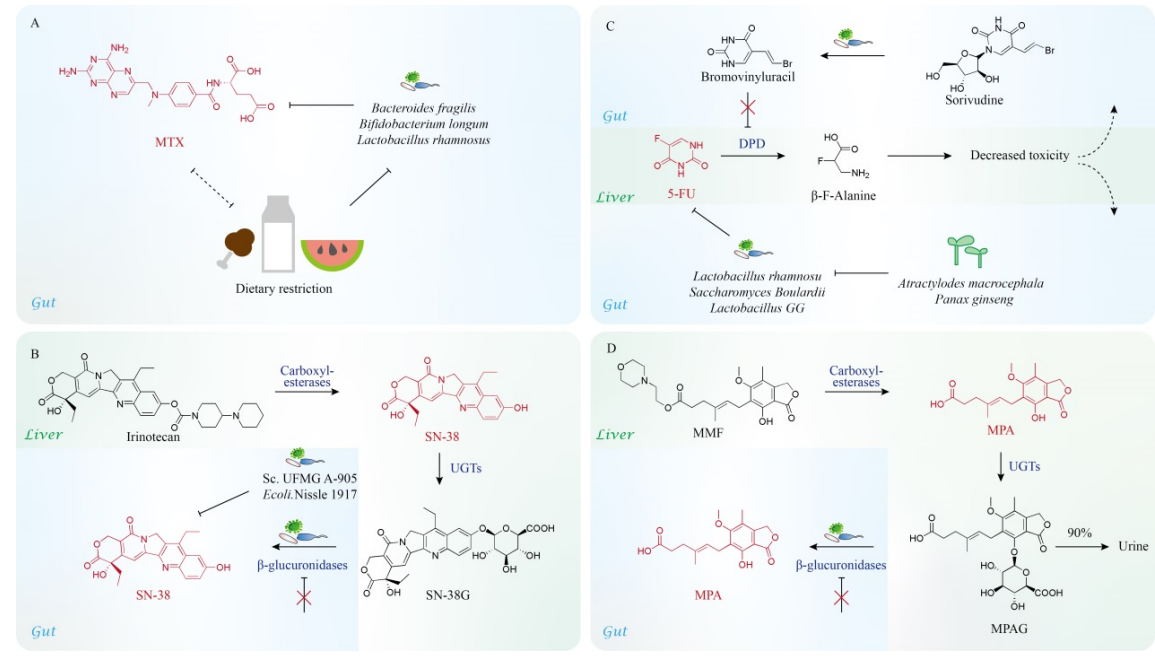

Fig.4 Drugs with gastrointestinal toxicity modulated by gut microbiota: methotrexate (A), irinotecan (B), 5fluorouracil (C), and mycophenolate mofetil (D). Red structures signify hepatotoxic drugs. Gastrointestinal toxicity of MPA and SN-38 can be attenuated by $\beta$-glucuronidases inhibition. Abbreviation: 5-FU, 5fluorouracil; DPD, dihydropyrimidine dehydrogenase; MMF, mycophenolate mofetil; MPA mycophenolic acid; MPAG, mycophenolic acid glucuronide; MTX, methotrexate; SN-38G, SN-38 glucuronide; UGTs, UDP-glucuronosyltransferases.

\subsection{Methotrexate}

Methotrexate (MTX) is an antifolic antineoplastic but brings about multiple side effects including hepatotoxicity, nephrotoxicity, pulmonary toxicity, and gastrointestinal toxicity, among which hepatotoxicity and gastrointestinal toxicity are the most common (Wang et al., 2018). In terms of gastrointestinal toxicity, it is characterized by gastrointestinal mucositis. A previous study found that MTX treatment substantially decreased gut microbial quantity and diversity of rats, with anaerobes and streptococci significantly decreased, which is proportionally associated with the severity of MTX-induced gastrointestinal mucositis (Fijlstra et al., 2015). A similar study confirmed the microbial dysbiosis caused by MTX in mice with a significantly decreased abundance of Bacteroides fragilis, and supplemental Bacteroides fragilis respited MTX-induced inflammatory reactions and macrophage polarization (Zhou et al., 2018a). Moreover, Bifidobacterium longum was also found to be a probiotic in attenuating MTX-induced intestine damage, which was discovered in the co-treatment of leucovorin with MTX for the sake of reducing its side effects (Huang et al., 2020b). Supplementation with probiotics perturbed by drugs is the most direct and explicit way in alleviating microbiota related adverse effects, but safety remains a question for long-term adhibition. In comparison, dietary control offers a safer alternative herein. It was found that dietary restriction remarkably enhanced the survival rate of mice exposed to a lethal dose of MTX and ameliorated MTX-induced intestine inflammation with Lactobacillus rhamnosusplaying a critical role (Tang et al., 2020). In this case, dietary restriction needless of specific diet, drug, or probiotics intervened achieved remarkable results in reducing MTX-induced gastrointestinal side effects, suggesting what we eat, which constitutes the major part of gastrointestinal contents, probably determines therapeutic and toxic outcomes of orally administered drugs somehow.

\subsection{Irinotecan}

Irinotecan (also called CPT-11) is one of the most commonly applied anticancer agents for colon carcinoma therapy but causes lethal toxicity in at least $36 \%$ of patients, most of whom undergo diarrhea, mucositis, and other gastrointestinal reactions (Encarnação et al., 2018). In the human body it is metabolized both by liver and gut microbiota (Fig.4): first converted into bioactive SN-38 by liver carboxylesterases and further transforms into SN-38G by conjugating with glucuronic acid catalyzed by hepatic UDP-glucuronosyltransferases 
(UGTs), which is then subject to gut bacterial $\beta$-glucuronidases to generate $\mathrm{SN}-38$, also a toxicant that induces damage of intestinal epithelial cells and severe diarrhea (Yang et al., 2018b). Due to the critical role of gut bacterial $\beta$-glucuronidases plays therein, it has been highlighted in mitigating irinotecan-induced gastrointestinal toxicity. Intestinal bacterial $\beta$-glucuronidases are considered to be a predictive biomarker of irinotecan triggered diarrhea severity (Chamseddine et al., 2019) and inhibition of it has been demonstrated to be effective in abrogating irinotecan toxicity (Wallace et al., 2010; Roberts et al., 2013). However, inhibition of bacterial $\beta$-glucuronidases of different origins and structures received distinctly different outcomes: inhibition of Firmicutes and Proteobacterium derived $\beta$-glucuronidases alleviated irinotecan-induced diarrhea in mice while inhibition ofBacteroidetes derived one did not, suggesting functional diversity in orthologous enzymes of gut microbiota (Wallace et al., 2015). Additionally, probiotics such as Saccharomyces cerevisiae UFMG A-905 (Bastos et al., 2016) and Escherichia coli Nissle 1917 (Wang et al., 2019c) treatment also has been proven to confer protective effects against irinotecan-induced gastrointestinal side effects.

\subsection{5-Fluorouracil}

5-Fluorouracil (5-FU), used for colorectal cancer therapy, is frequently related to mucosal side effects such as diarrhea and mucositis. 5-FU in the human body is mainly detoxified by hepatic dihydropyrimidine dehydrogenase (DPD), inhibition of which induces 5-FU accumulation and consequently aggravates adverse effects. In 1993, a combination of Sorivudine and 5-FU resulted in 18 deaths with symptoms of diarrhea and decreased blood cells and platelets in Japan, 40 days after Sorivudine was approved in clinical use. It was found that gut microbial metabolite of Sorivudine, bromovinyluracil inactivated DPD and thus limited detoxification of 5-FU (Klaassen and Cui, 2015). It is an example that gut microbiota mediates drug-drug interactions and further studies illustrate that gut microbiota regulates 5-FU toxicity directly. A study relating 5-FU-induced colon inflammation to gut microbiota suggested that 5-FU caused alterations in gut microbiota and fecal transplantation from 5-FU treated mice to normal ones diminished functional performance and altered colon inflammatory markers of the latter (Sougiannis et al., 2019). Another study focusing on fecal transplantation from the therapeutic side revealed transplantation of fecal microbiota from health donor mice attenuated 5-FU-based chemotherapy regimen, FOLFOX (5-FU, leucovorin, and oxaliplatin)induced intestinal mucositis by downregulating expression levels of toll-like receptors (TLRs), MyD88, and serum IL-6 with verified safety (Chang et al., 2020a). Similarly, probiotics were also found to palliate 5FU-triggered intestinal injury, including Lactobacillus casei rhamnosu (Chang et al., 2018), Saccharomyces Boulardii (Justino et al., 2014), and LactobacillusGG (Holma et al., 2013), in animal experiments and human trials respectively. The human trial also showed that methanogenic archaea made a difference in whether patients underwent diarrhea or constipation after 5-FU treatment (Holma et al., 2013). Additionally, prebiotics such as the herbal pair, Atractylodes Macrocephala and Panax Ginseng conferred a protective effect against 5-FU by restoring gut microbiota disturbed by it and especially inhibited 5-FU-induced release of intestinal inflammatory cytokines (Wang et al., 2019a). As we put, strategies for tackling 5-FU-induced diarrhea and mucositis are restricted within conventional ones, mainly including fecal microbial transplant, probiotics, and prebiotics supplementation. The discovery of intestinal microbial metabolism of the drug proffers different and effective methods in coping with its toxicity, suggesting microbial metabolism of drugs plays a significant role in determining toxic outcomes of drugs.

\subsection{Mycophenolate mofetil}

Mycophenolate mofetil (MMF) is an immunosuppressive agent prescribed to patients after transplantation and also exerts severe gastrointestinal side effects which limit its application. As a prodrug, it is hydrolyzed to mycophenolic acid (MPA) to confer efficacy and is converted into glucuronidated MPA (MPAG) by hepatic enzymes. MPAG is primarily excreted via urine but approximately $10 \%$ of it is transported into the gastrointestinal tract and is metabolized by gut microbial ßglucuronidase back into MPA (Fig.4), whose accumulation in the colon is associated with MMF-induced colonic inflammation (Lamba et al., 2014). A study revealed that MMF disturbed gut microbiota of which Proteobacteria (specifically Escherichia/Shigella) and gene expression of LPS biosynthesis were significantly enhanced while overall diversity was diminished in mice, which could be reversed by broad-spectrum antibiotics treatment (Flannigan et al., 2018). Consistent with 
the metabolic pathway of MPA in the gut, it was found that MMF induced active $\beta$ glucuronidase expression and in turn exacerbated its gastrointestinal adverse effect, which could be eliminated by vancomycin (Taylor et al., 2019), suggesting that $\beta$ glucuronidase also can be an efficient target in alleviating MMF toxicity.

\section{GUT MICROBIOTA-MEDIATED DRUG NEUROTOXICITY}

The intestinal microbiota produces vast amounts of metabolites some of which exert neurotoxicity, such as D-lactic acid and ammonia (Galland, 2014). Ammonia is a key factor contributing to hepatic encephalopathy which is accumulated in the brain when the liver undergoes dysfunction (Wijdicks, 2016), and modulation of gut microbiota has been demonstrated effective in decreasing ammonia production and subsequently alleviating encephalopathy (Shen et al., 2015). Moreover, the intestinal microbiota is also indirectly engaged in neurotoxicity of xenobiotics including environmental neurotoxicants, metals, drugs, and pesticides (Dempsey et al., 2019) of which neurotoxicity is the most frequently encountered adverse effect in antibiotic exposure representing in multiple ways including headache, delirium, psychosis, and seizure (Bangert and Hasbun, 2019; Champagne-Jorgensen et al., 2019) (Fig.5). While some absorbable antibiotics enter the brain through blood brain barrier (BBB) and cause direct damage, most non-absorbable antibiotics such as neomycin and vancomycin also induce impairments in nervous system and behavior, suggesting a pivotal of the gut-brain axis plays therein (Fröhlich et al., 2016). With antibiotic treatment proven efficient in a variety of microbiotaimplicated diseases, tackling antibiotics-induced neurotoxicity seems more troublesome, especially with its mechanisms remaining ambiguous. Besides antibiotics, anesthetics and psychotropic drugs are also found to exert neurotoxicity with intestinal microbiome involved and they will also be discussed afterward.

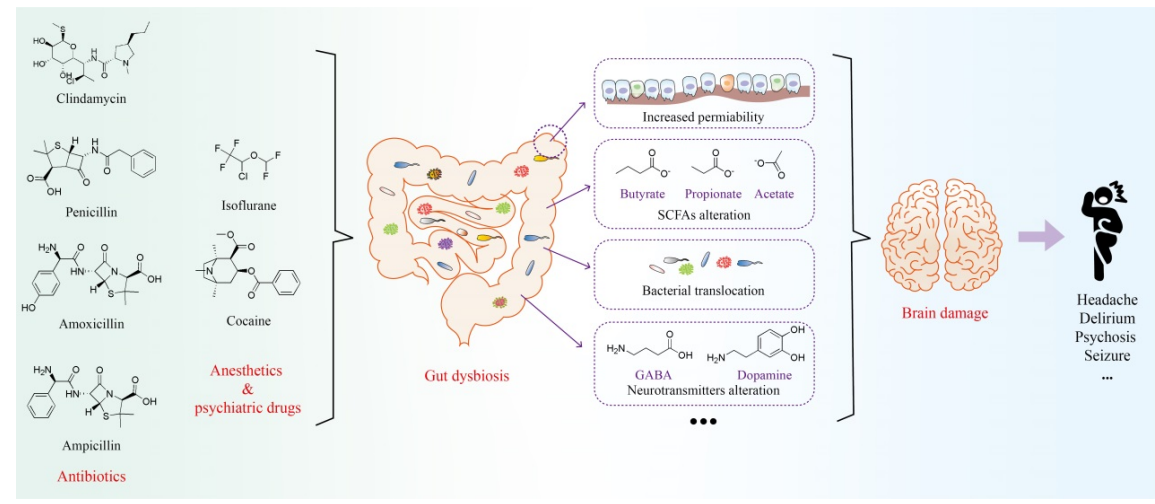

Fig.5 Drugs with neurotoxicity modulated by gut microbiota and possible mechanisms. Abbreviations: GABA, $\gamma$-amino butyric acid; SCFAs, short-chain fatty acids.

\subsection{Antibiotics}

Neurological disorders are usually seen in antibiotics-exposed children who are represented with autism spectrum disorders (ASD). Bacteria overgrowth and especially an abundance of Bacteroidetes andClostridium phyla are closely related to ASD, with the former one specializing in producing propionate and LPS and the latter one propionate. Such opportunistic pathogenic microbiome like clostridial spores are often resistant to most antibiotics and since antibiotics eliminate the majority of intestinal bacteria, it consequently leaves pathogenic bacteria a higher proportion which may be one of the mechanisms that antibiotics induce autism in children (Finegold, 2008). Indeed, both propionic acid and clindamycin administrations induced neurotoxicity in the rodent model and the clindamycin-induced neurotoxicity might be partly attributed to an overgrowth of propionic acid-producing bacteria (El-Ansary et al., 2013). Additionally, both of them depleted $\mathrm{Mg}^{2+}$ and GABA and increased $\mathrm{Na}^{+} / \mathrm{Mg}^{2+}$ and glutamate/GABA ratios in the brain which could be restored by replenishing probiotics such asBifidobacteria , Lactobacilli and ProtexinR (Al-Orf et al., 2018; El-Ansary et al., 2018). An experiment conducted in mice of late gestation suggested that a low dose of exposure to penicillin induced gender-specific aberrant behaviors in fetus mice with male mice displaying 
more anxiety-like behaviors which were associated with altered expression of arginine vasopressin receptor 1A/B (AVPR1A/B) and oxytocin receptor (OXTR), imbalanced forkhead box P3-positive $\left(\mathrm{FOXP}^{+}\right) \mathrm{T}_{\text {reg }}$ cells and gut microbial dysbiosis (Champagne-Jorgensen et al., 2020). A similar study conducted in perinatal mice revealed that long-term administration of low-dose penicillin led to changes in gut microbiota, intestinal barrier function, BBB integrity, cytokines expression, and behavior while supplementation of Lactobacillus rhamnosus JB-1 showed partial preventive effects against these symptoms (Leclercq et al., 2017). Disturbance of gut microbiots by antibiotics in an early age tends to alter neurodevelopment which may lead to functional bowel disorders later in life (Caputi et al., 2017). Another study also found that amoxicillin abuse caused gut microbiota dysbiosis and histopathological and neurobehavioral alterations in adult mice, which may explain the rising trend of neuropsychiatric diseases in antibiotics abusing countries (Helaly et al., 2019). Comparing the studies and findings, we find that antibiotic exposure leads to neurotoxicity in fetuses with a low dosage while antibiotic abuse in adults may also induce nerve injury, urging for prudent usage of antibiotics. Besides, ampicillin was also discovered to confer neurotoxicity with modulation of gut microbiota effective in attenuating it. The neurotoxic effect of ampicillin was linked with an increase in lipid peroxidation and a decrease in GSH and potassium levels, and alteration in neurotransmitters which could be reversed by diet intervention, partly due to restored gut microbiota (El-Ansary et al., 2015; Bhat et al., 2016). Antibiotics have been used as a valuable tool in microbiota-related studies but their neurotoxicity has been long neglected in the process (Champagne-Jorgensen et al., 2019). While the direct nerve damage caused by absorbable antibiotics is widely recognized, neurotoxicity of non-absorbable antibiotics seems unintelligible. Given the emerging importance of microbiota-gut-brain axis, it is reasonable to connect antibiotic neurotoxicity with gut microbiota, which has been demonstrated by some researches mentioned above. However, these phenomenon-based studies fail to decipher incisive interactions between antibiotics and neurotoxicity and it can be improved with the uncovering mechanisms of gut-brain axis.

\subsection{Anesthetics and psychiatric drugs}

Besides antibiotics, some anesthetic agents also induce neurological injuries in neonates. Evidence suggests that inhaled isoflurane is neurotoxic to developing brains of neonatal rodents which may be associated with disturbed gut microbial composition (Wang et al., 2019b). The isoflurane inhaled rats displayed gut dysbiosis with an abundance of Firmicutes, Proteobacteria,Clostridia, Clostridiales , and Lachnospiraceaesignificantly increased and Bacteroidetes, Actinobacteria, Bacteroidia, and Bacteroidaceae decreased. However, it is a preliminary study connecting anesthetic neurotoxicity to intestinal microbiota and further research should be conducted. What is similar herein is volatilized cocaine whose cause of brain functional and emotional disorder was partly attributed to gut dysbiosis (Scorza et al., 2019).

\subsection{Substances other than drugs}

Some environmental toxicants especially pesticides represented by organophosphates affect the central nervous system badly and their interactions with gut microbiota have been elaborately elucidated with epithelial permeability, bacterial translocation, and other intestinal microbial factors found to contribute to their neurotoxicity (Roman et al., 2019). Acetylcholinesterase (AChE) inhibition is a common cause of neurotoxicity of these kinds of chemicals but with gut microbiota emerging, alterations in intestinal neurotransmitters and metabolites are also frequently found to contribute to neurotoxicity of them (Lin et al., 2020). Gut microbial metabolism also plays an instrumental role in organophosphates as neurotoxicity of chlorpyrifos was exacerbated after transformation to chlorpyrifos-oxon by gut-derived Lactobacillus plantarum, and could be attenuated with antibiotics and probiotics treatments (Daisley et al., 2018). Rotenone, another kind of pesticides, was used to establish a PD model in animals and it was found that probiotics could rescue dopaminergic neurons in the PD model by promoting butyrate production (Srivastav et al., 2019). Moreover, prebiotics like fructo- and xylo-oligosaccharides also conferred protective effects against neurotoxic chemicals by restoring the activity of $\mathrm{AChE}$ and improving levels of dopamine in the maternal cortex (Krishna and Muralidhara, 2018). These researches and discoveries may proffer references for mechanistic investigations of drug-related neurotoxicity.

\section{CONNECTING THE DOTS: GUT-LIVER-BRAIN AIXS IN DRUG TOXICITY}


Patients with chronic liver diseases and cirrhosis often present with global mucosal immune impairment and it can progress with advancing degrees of cirrhosis and further induces HE (Bajaj and Khoruts, 2020). HE is a major complication of liver diseases that can be initiated by a wide spectrum of factors including systemic inflammation, liver cirrhosis, microbiota alteration, skeletal muscle disability, and medications (Bajaj, 2018). As we mentioned above, ammonia is a significant contributor to HE: normally, the ammonia in the gut lumen is transferred into the liver where it is metabolized into urea via urea cycle but when the host undergoes hyperammonemia or liver diseases, it enters the brain and causes aberrantly functioning neurons (Fig.2) (Wijdicks, 2016). It is a representative example of how the gut, liver, and brain are functionally connected and modulations of gut microbiota by supplementing prebiotics (lactulose) and probiotics, administering antibiotics (rifaximin), altering the dietary structure, and FMT are emerging in HE treatment (Amodio et al., 2014; Campion et al., 2019; Weir, 2020). What accords with the theme here is that HE can be triggered by medications. Although alterations in the brain caused by APAP have long been considered secondary effects of acute liver injury, recently it is found to be a cannabinoid system modulator and cause "in situ" toxicity in the brain especially at high doses (Ghanem et al., 2016). Acute APAP intoxication disturbs neurotransmitters including GABA, glutamate, dopamine, serotonin, norepinephrine, and acetylcholine, increases oxidative stress and reactive astrogliosis in the brain, and consequently induces HE (Saad et al., 2018; Vigo et al., 2019). And similar to the case in antibiotics, maternal use of APAP also induces neurofunctional impairments in the progeny (Klein et al., 2020). In addition to the fact that APAP also distributes and is metabolized in the brain which directly affects brain function, the gut-brain axis may play a part therein, and the liver may be an important mediator. Carbon tetrachloride $\left(\mathrm{CCl}_{4}\right)$ is one of the most commonly used chemicals in establishing liver injury models in animals. It is reported that $\mathrm{CCl}_{4}$ also can be utilized to establish $\mathrm{HE}$ model in rats and the $\mathrm{CCl}_{4}$-treated rats showed $\mathrm{HE}$ symptoms of impaired spontaneous movement, cachexia, somnolence, and brain histological alterations (Wang et al., 2017). FMT improved hepatic necrosis, intestinal mucosal barrier damage, behaviors, HE grade, and spatial learning capability of these rats (Wang et al., 2017), revealing that the gut microbiota can be a common target in treating liver and brain diseases. Besides, drugs such as 5-FU that induce hyperammonemia also may cause severe HE and should be given special attention in clinical use (Chahin et al., 2020). Theoretically, drugs eliciting hepatotoxicity or inducing hyperammonemia are potential in causing HE. Besides mechanisms mentioned in drug hepatotoxicity, possible mechanisms involved therein may include enhancing ammonia-producing microbiota, altering neurotransmitters, and increasing gut permeability. Extendedly speaking, drugs that induce gut dysbiosis tend to more or less impact the organs connected by the gut. The gut-liver-brain axis may explain the multi-facet toxicity property of some drugs, foresee probable toxicological responses in other organs besides conventional ones, and offer countermeasures in coping with them.

\section{CONCLUSIONS AND PERSPECTIVES}

Gut microbiota increasingly appears in our field of view whether as a pathogeny or remedy in recent decades. It interacts with almost whatever enters the intestinal tract and directly or indirectly influences fates of xenobiotics. Oral drugs are especially within the scope of gut microbial action with their therapeutic and toxic outcomes largely determined by it. In drug-related hepatotoxicity and gastrointestinal toxicity, gut microbiota acts more like a metabolic role with some drugs exhibiting attenuated toxicity after gut microbial metabolism while some in contrast. Within the process, microbial $\beta$ glucuronidase plays a crucial role. Bglucuronidase is a hydrolase extensively distributed in mammalian tissues, body fluids, and microbiota. A wide range of toxic drugs undergo detoxification in the liver by UGTs but the glucuronic acid conjugated metabolites are reabsorbed in the gut where gut microbial $\beta$ glucuronidase transforms the metabolites back into their toxic prototype. Inhibition of ßglucuronidase activity has been demonstrated effective in alleviating drug toxicities and it presents as an attractive target for the development of novel therapeutics. Some natural products such as quercetin can not only generate protective metabolites by gut microbiota but also function as ßglucuronidase inhibitors. However, the clinical utilization of currently discovered $\beta$ glucuronidase inhibitors is limited by poor pharmacokinetic profile (Awolade et al., 2020). Still and all, ßglucuronidase may serve as a potent target in respiting drug toxicity. Besides, the use of antibiotics and pre-/probiotics is also effective in coping with drug toxicity. Although gut microbiota are highly dynamic, the diminishing of opportunistic 
microbiota and supplementation of beneficial ones are truly effective in attenuating relevant diseases as well as drug toxicity. It is worth noting that antibiotics also cause severe neurotoxicity which is associated with gut microbiota. Propionic acid is an important factor that contributes to autistic symptoms in children with antibiotics usage and it can be decreased by modulating gut microbiota. It also hints us that utilization of antibiotics is a double-edged sword: for one thing, targeting gut microbiota by using antibiotics has become a rising field in liver disease treatment; for another, neurotoxicity of antibiotics mediated by gut microbiota should beware. On the whole, gut microbiota is a common target for some drugs that cause hepatotoxicity, gastrointestinal toxicity, and neurotoxicity. Moreover, we come to a hypothesis that drugs capable of inducing gut dysbiosis tend to more or less impact the gut-connected organs as evidenced by the drug-induced HE, which means there is an underlying link among the gut, liver, brain, and possible other organs in drug-induced toxicity.

Table 1 Drugs with toxicity modulated by gut microbiota and intervening strategies

\begin{tabular}{|c|c|c|c|c|}
\hline Drugs & Toxicity & Interventions & Toxic effects & Mechanism \\
\hline \multirow[t]{5}{*}{$\overline{\mathrm{APAP}}$} & Hepatotoxicity & $p$-cresol & - & Competes for $\mathrm{s}$ \\
\hline & & $\mathrm{PPD}$ & - & Depletes glutath \\
\hline & & DOPA, 4-HPA & - & Activate Nrf2 an \\
\hline & & Fructose & - & Alters gut mic \\
\hline & & Bacillus spore & - & Alters proinfla \\
\hline \multirow[t]{2}{*}{ Tacrine } & Hepatotoxicity & $\beta$-glucuronidase & - & Increases toxic \\
\hline & & Antibiotics & - & Decreases toxi \\
\hline \multirow[t]{2}{*}{ Triptolide } & Hepatotoxicity & Antibiotics & - & Increases toxic \\
\hline & & Propionate & - & - \\
\hline Geniposide & Hepatotoxicity & Antibiotics & - & Decreases toxic \\
\hline \multirow[t]{4}{*}{ MTX } & Gastrointestinal toxicity & Bacteroides fragilis & - & Restores gut $\mathrm{m}$ \\
\hline & & Bifidobacterium longum & - & \\
\hline & & Lactobacillus rhamnosus & - & \\
\hline & & Dietary restriction & - & \\
\hline \multirow[t]{3}{*}{ Irinotecan } & Gastrointestinal toxicity & $\beta$-glucuronidase inhibition & - & Decreases toxi \\
\hline & & Saccharomyces cerevisiae UFMG A-905 & - & Restores gut $\mathrm{n}$ \\
\hline & & Escherichia coli Nissle 1917 & - & \\
\hline \multirow[t]{6}{*}{$5-\mathrm{FU}$} & Gastrointestinal toxicity & Sorivudine & - & Inactivates meta \\
\hline & & FMT & - & Downregulates \\
\hline & & Lactobacillus casei rhamnosu & - & Restore gut $\mathrm{m}$ \\
\hline & & Saccharomyces Boulardii & - & \\
\hline & & Lactobacillus GG & - & \\
\hline & & Atractylodes Macrocephala and Panax Ginseng & - & \\
\hline MMF & Gastrointestinal toxicity & Antibiotics & - & Decreases toxic \\
\hline \multirow[t]{3}{*}{ Clindamycin } & Neurotoxicity & - & - & Overgrowth of \\
\hline & & Bifidobacteria, Lactobacilli & - & Restore $\mathrm{Mg} 2+$ \\
\hline & & ProtexinR & - & \\
\hline \multirow[t]{3}{*}{ Penicillin } & Neurotoxicity & - & - & Alters expressior \\
\hline & & - & - & Changes gut mic \\
\hline & & Lactobacillus rhamnosus JB-1 & - & Restore gut mi \\
\hline Amoxicillin & Neurotoxicity & - & - & Gut microbiot \\
\hline \multirow[t]{3}{*}{ Ampicillin } & Neurotoxicity & - & - & Increases lipid $p$ \\
\hline & & - & - & Alters neurotra \\
\hline & & Dietary intervention & - & Restore gut mi \\
\hline Isoflurane & Neurotoxicity & - & - & Gut microbiota \\
\hline Cocaine & Neurotoxicity & - & - & Gut microbiota \\
\hline
\end{tabular}


Abbreviations: 4-HPA, 4-hydroxyphenylacetic acid; 5-FU, 5-fluorouracil; APAP, acetaminophen; AVPR1A/B, arginine vasopressin receptor 1A/B; BBB, blood brain barrier; DOPA, 3,4dihydroxyphenylacetic acid; FMT, fecal microbiota transplantation; $\mathrm{FOXP}^{+}$, forkhead box P3-positive; GABA, $\gamma$-aminobutyric acid; GSH, glutathione; MMF, mycophenolate mofetil; MTX, methotrexate; OXTR, oxytocin receptor; PPD, 1-phenyl-1,2-propanedione; TLRs, toll-like receptors.

- signifies that toxicity of drugs is enhanced after intervention and - signifies that toxicity of drugs is decreased after intervention.

In conclusion, gut microbiota modulates drug toxicity within the following aspects (Table 1): (a) gut-derived endogenous metabolites inhibit drug metabolism by competitive effects and lead to drug accumulation; (b) gut-derived endogenous and exogenous metabolites restore gut dysbiosis caused by drugs and ameliorate drug toxicity; (c) specific gut microbial enzymes transform drugs into toxic or nontoxic metabolites; (d) prebiotics, probiotics, and diet intervention attenuate drug toxicity by regulating gut microbiota; (e) gut microbial metabolites inactivate metabolic enzymes of drugs and also lead to drug accumulation; (f) gut microbiota translocation induced by drugs causes damages especially in the brain. Currently, high-throughput technologies such as sequencing analysis and omics technologies facilitate the unprecedented development of mechanistic studies of gut microbiota. In terms of drug toxicity, special attention should be given to drug metabolisms both in the liver and gut, which appeals for development in analytical methods. The utilization of gut microbiota as biomarkers of diseases and guidance for personalized medicine is a trend of the future.

Declarations of interest : None.

Acknowledgements : This work was supported by National Natural Science Foundation of China (grant number: 81973457).

\section{References :}

Aabed, K., Bhat, R.S., Al-Dbass, A., Moubayed, N., Algahtani, N., Merghani, N.M., et al. (2019). Bee pollen and propolis improve neuroinflammation and dysbiosis induced by propionic acid, a short chain fatty acid in a rodent model of autism. Lipids Health Dis. $18: 1-8$.

Aburada, M., and Kobashi, K. (1994). Enzymic Studies on the Animal and Intestinal Bacterial Metabolism of Geniposide. Biol. Pharm. Bull.

Al-Orf, N., El-Ansary, A., Bjørklund, G., Moubayed, N., Bhat, R.S., and Bacha, A. Ben (2018). Therapeutic effects of probiotics on neurotoxicity induced by clindamycin and propionic acid in juvenile hamsters. Metab. Brain Dis. 33 : 1811-1820.

Amodio, P., Canesso, F., and Montagnese, S. (2014). Dietary management of hepatic encephalopathy revisited. Curr. Opin. Clin. Nutr. Metab. Care17 : 448-452.

Aron-Wisnewsky, J., Warmbrunn, M., Nieuwdorp, M., and Clément, K. (2020). Nonalcoholic Fatty Liver Disease: Modulating Gut Microbiota to Improve Severity? Gastroenterology.

Awolade, P., Cele, N., Kerru, N., Gummidi, L., Oluwakemi, E., and Singh, P. (2020). Therapeutic significance of $\beta$-glucuronidase activity and its inhibitors: A review. Eur. J. Med. Chem.

Bajaj, J.S. (2018). Hepatic encephalopathy: classification and treatment. J. Hepatol. 68 : 838-839.

Bajaj, J.S., and Khoruts, A. (2020). Microbiota changes and intestinal microbiota transplantation in liver diseases and cirrhosis. J. Hepatol. 72 : 1003-1027.

Bangert, M.K., and Hasbun, R. (2019). Neurological and Psychiatric Adverse Effects of Antimicrobials. CNS Drugs.

Bastos, R.W., Pedroso, S.H.S.P., Vieira, A.T., Moreira, L.M.C., França, C.S., Cartelle, C.T., et al. (2016). Saccharomyces cerevisiae UFMG A-905 treatment reduces intestinal damage in a murine model of irinotecaninduced mucositis. Benef. Microbes. 
Bethesda (MD): National Institute of Diabetes and Digestive and Kidney Diseases (2018). LiverTox: Clinical and Research Information on Drug- Induced Liver Injury.

Bhat, R.S., Chandrul, K.K., and El-Ansary, A. (2016). Beneficial effects of a protein rich diet on coping neurotransmitter levels during ampicillin-induced neurotoxicity compared to propionic-acid induced autistic biochemical features. Int. J. Mol. Cell. Med.

Budzyński, J., and Kłopocka, M. (2014). Brain-gut axis in the pathogenesis of Helicobacter pylori infection. World J. Gastroenterol.20 : 5212-5225.

Campion, D., Giovo, I., Ponzo, P., Saracco, G.M., Balzola, F., and Alessandria, C. (2019). Dietary approach and gut microbiota modulation for chronic hepatic encephalopathy in cirrhosis. World J. Hepatol.11 : 489-512.

Caputi, V., Marsilio, I., Filpa, V., Cerantola, S., Orso, G., Bistoletti, M., et al. (2017). Antibiotic-induced dysbiosis of the microbiota impairs gut neuromuscular function in juvenile mice. Br. J. Pharmacol.

Chahin, M., Krishnan, N., Chhatrala, H., and Shaikh, M. (2020). A 5-Fluorouracil-Induced Hyperammonemic Encephalopathy Challenged with Capecitabine. Case Rep. Oncol. Med.

Champagne-Jorgensen, K., Kunze, W.A., Forsythe, P., Bienenstock, J., and McVey Neufeld, K.A. (2019). Antibiotics and the nervous system: More than just the microbes? Brain. Behav. Immun. 777 : 7-15.

Champagne-Jorgensen, K., Mian, M.F., Kay, S., Hanani, H., Ziv, O., McVey Neufeld, K.A., et al. (2020). Prenatal low-dose penicillin results in long-term sex-specific changes to murine behaviour, immune regulation, and gut microbiota. Brain. Behav. Immun. 84: 154-163.

Chamseddine, A.N., Ducreux, M., Armand, J.P., Paoletti, X., Satar, T., Paci, A., et al. (2019). Intestinal bacterial $\beta$-glucuronidase as a possible predictive biomarker of irinotecan-induced diarrhea severity. Pharmacol. Ther. 199 : $1-15$.

Chang, C.W., Lee, H.C., Li, L.H., Chiau, J.S.C., Wang, T.E., Chuang, W.H., et al. (2020a). Fecal microbiota transplantation prevents intestinal injury, upregulation of toll-like receptors, and 5-fluorouracil/oxaliplatininduced toxicity in colorectal cancer. Int. J. Mol. Sci. 21 :.

Chang, C.W., Liu, C.Y., Lee, H.C., Huang, Y.H., Li, L.H., Chiau, J.S.C., et al. (2018). Lactobacillus casei Variety rhamnosus probiotic preventively attenuates 5-Fluorouracil/Oxaliplatin-induced intestinal injury in a syngeneic colorectal cancer model. Front. Microbiol.9 : 1-15.

Chang, J., Wang, T., Wang, P., Yin, Q., Liu, C., Zhu, Q., et al. (2020b). Compound probiotics alleviating aflatoxin B1 and zearalenone toxic effects on broiler production performance and gut microbiota. Ecotoxicol. Environ. Saf.

Chang, L., Xu, D., Zhu, J., Ge, G., Kong, X., and Zhou, Y. (2020c). Herbal Therapy for the Treatment of Acetaminophen-Associated Liver Injury: Recent Advances and Future Perspectives. Front. Pharmacol.11 : $1-13$.

Chao, X., Wang, H., Jaeschke, H., and Ding, W.X. (2018). Role and mechanisms of autophagy in acetaminophen-induced liver injury. Liver Int. $38: 1363-1374$.

Chen, Z., Zhou, D., Han, S., Zhou, S., and Jia, G. (2019). Hepatotoxicity and the role of the gut-liver axis in rats after oral administration of titanium dioxide nanoparticles. Part. Fibre Toxicol.

Chi, L., Xue, J., Tu, P., Lai, Y., Ru, H., and Lu, K. (2019a). Gut microbiome disruption altered the biotransformation and liver toxicity of arsenic in mice. Arch. Toxicol.

Chi, Y., Lin, Y., Lu, Y., Huang, Q., Ye, G., and Dong, S. (2019b). Gut microbiota dysbiosis correlates with a low-dose PCB126-induced dyslipidemia and non-alcoholic fatty liver disease. Sci. Total Environ. 
Cho, S., Tripathi, A., Chlipala, G., Green, S., Lee, H., Chang, E.B., et al. (2017). Fructose diet alleviates acetaminophen-induced hepatotoxicity in mice. PLoS One 12:1-19.

Choi, J.S., Han, Y.R., Byeon, J.S., Choung, S.Y., Sohn, H.S., and Jung, H.A. (2015). Protective effect of fucosterol isolated from the edible brown algae, Ecklonia stolonifera and Eisenia bicyclis, on tert-butyl hydroperoxide- and tacrine-induced HepG2 cell injury. J. Pharm. Pharmacol.

Ciorba, M.A., Hallemeier, C.L., Stenson, W.F., and Parikh, P.J. (2015). Probiotics to prevent gastrointestinal toxicity from cancer therapy: An interpretive review and call to action. Curr. Opin. Support. Palliat. Care $9: 157-162$.

Clayton, T.A., Baker, D., Lindon, J.C., Everett, J.R., and Nicholson, J.K. (2009). Pharmacometabonomic identification of a significant host-microbiome metabolic interaction affecting human drug metabolism. Proc. Natl. Acad. Sci. U. S. A. 106 : 14728-14733.

Coryell, M., McAlpine, M., Pinkham, N. V., McDermott, T.R., and Walk, S.T. (2018). The gut microbiome is required for full protection against acute arsenic toxicity in mouse models. Nat. Commun.

Cryan, J.F., O'riordan, K.J., Cowan, C.S.M., Sandhu, K. V., Bastiaanssen, T.F.S., Boehme, M., et al. (2019). The microbiota-gut-brain axis. Physiol. Rev. 99 : 1877-2013.

Daisley, B.A., Trinder, M., McDowell, T.W., Collins, S.L., Sumarah, M.W., and Reid, G. (2018). Microbiotamediated modulation of organophosphate insecticide toxicity by species-dependent interactions with lactobacilli in a Drosophila melanogaster insect model. Appl. Environ. Microbiol. 84 :.

Dempsey, J.L., Little, M., and Cui, J.Y. (2019). Gut microbiome: An intermediary to neurotoxicity. Neurotoxicology $75: 41-69$.

Dey, P. (2019). Gut microbiota in phytopharmacology: A comprehensive overview of concepts, reciprocal interactions, biotransformations and mode of actions. Pharmacol. Res. 147 : 104367.

Dinan, T.G., and Cryan, J.F. (2017). Brain-Gut-Microbiota Axis and Mental Health. Psychosom. Med.

El-Ansary, A., Bacha, A. Ben, Bjørklund, G., Al-Orf, N., Bhat, R.S., Moubayed, N., et al. (2018). Probiotic treatment reduces the autistic-like excitation/inhibition imbalance in juvenile hamsters induced by orally administered propionic acid and clindamycin. Metab. Brain Dis. 33 : 1155-1164.

El-Ansary, A., Bhat, R.S., Al-Daihan, S., and Dbass, A.M. Al (2015). The neurotoxic effects of ampicillinassociated gut bacterial imbalances compared to those of orally administered propionic acid in the etiology of persistent autistic features in rat pups: Effects of various dietary regimens. Gut Pathog.

El-Ansary, A., Shaker, G.H., El-Gezeery, A.R., and Al-Ayadhi, L. (2013). The neurotoxic effect of clindamycin - Induced gut bacterial imbalance and orally administered propionic acid on DNA damage assessed by the comet assay: Protective potency of carnosine and carnitine. Gut Pathog. 5 : 1-10.

El-Nezami, H.S., Polychronaki, N.N., Ma, J., Zhu, H., Ling, W., Salminen, E.K., et al. (2006). Probiotic supplementation reduces a biomarker for increased risk of liver cancer in young men from Southern China. Am. J. Clin. Nutr.

Encarnação, J.C., Pires, A.S., Amaral, R.A., Gonçalves, T.J., Laranjo, M., Casalta-Lopes, J.E., et al. (2018). Butyrate, a dietary fiber derivative that improves irinotecan effect in colon cancer cells. J. Nutr. Biochem.

Ervin, S.M., Ramanan, S.V., and Bhatt, A.P. (2020). Relationship Between the Gut Microbiome and Systemic Chemotherapy. Dig. Dis. Sci. $65: 874-884$.

Fader, K.A., Nault, R., Zhang, C., Kumagai, K., Harkema, J.R., and Zacharewski, T.R. (2017). 2,3,7,8Tetrachlorodibenzo-p-dioxin (TCDD)-elicited effects on bile acid homeostasis: Alterations in biosynthesis, enterohepatic circulation, and microbial metabolism. Sci. Rep. 7:1-17. 
Fang, X. (2019). Microbial treatment: the potential application for Parkinson's disease. Neurol. Sci. 40 : $51-58$.

Fijlstra, M., Ferdous, M., Koning, A.M., Rings, E.H.H.M., Harmsen, H.J.M., and Tissing, W.J.E. (2015). Substantial decreases in the number and diversity of microbiota during chemotherapy-induced gastrointestinal mucositis in a rat model. Support. Care Cancer 23 : 1513-1522.

Finegold, S.M. (2008). Therapy and epidemiology of autism-clostridial spores as key elements. Med. Hypotheses.

Fiori, J., Turroni, S., Candela, M., and Gotti, R. (2020). Assessment of gut microbiota fecal metabolites by chromatographic targeted approaches. J. Pharm. Biomed. Anal. 1777 : 112867.

Flannigan, K.L., Taylor, M.R., Pereira, S.K., Rodriguez-Arguello, J., Moffat, A.W., Alston, L., et al. (2018). An intact microbiota is required for the gastrointestinal toxicity of the immunosuppressant mycophenolate mofetil. J. Hear. Lung Transplant. 37 : 1047-1059.

Fröhlich, E.E., Farzi, A., Mayerhofer, R., Reichmann, F., Jačan, A., Wagner, B., et al. (2016). Cognitive impairment by antibiotic-induced gut dysbiosis: Analysis of gut microbiota-brain communication. Brain. Behav. Immun.

Galland, L. (2014). The gut microbiome and the brain. J. Med. Food17 : 1261-1272.

Ghanem, C.I., Pérez, M.J., Manautou, J.E., and Mottino, A.D. (2016). Acetaminophen from liver to brain: New insights into drug pharmacological action and toxicity. Pharmacol. Res.

Gong S, Lan T, Zeng L, Luo H, Yang X, Li N, Chen X, Liu Z, Li R, Win S, Liu S, Zhou H, Schnabl B, Jiang Y, Kaplowitz N, C.P. (2018). Gut microbiota mediates diurnal variation of acetaminophen induced acute liver injury in mice. J. Hepatol. $69: 51-59$.

Gong, X., Li, X., Bo, A., Shi, R.Y., Li, Q.Y., Lei, L.J., et al. (2020). The interactions between gut microbiota and bioactive ingredients of traditional Chinese medicines: A review. Pharmacol. Res. 157 : 104824.

Gopalakrishnan, V., Helmink, B.A., Spencer, C.N., Reuben, A., and Wargo, J.A. (2018). The Influence of the Gut Microbiome on Cancer, Immunity, and Cancer Immunotherapy. Cancer Cell.

Gori, S., Inno, A., Belluomini, L., Bocus, P., Bisoffi, Z., Russo, A., et al. (2019). Gut microbiota and cancer: How gut microbiota modulates activity, efficacy and toxicity of antitumoral therapy. Crit. Rev. Oncol. Hematol.

Gorkiewicz, G., and Moschen, A. (2018). Gut microbiome: a new player in gastrointestinal disease. Virchows Arch.

Hartmann, P., Chu, H., Duan, Y., and Schnabl, B. (2019). Gut microbiota in liver disease: Too much is harmful, nothing at all is not helpful either. Am. J. Physiol. - Gastrointest. Liver Physiol.

Helaly, A.M.N., El-Attar, Y.A., Khalil, M., Ahmed Ghorab, D.S.E.-D., and El- Mansoury, A.M. (2019). Antibiotic Abuse Induced Histopathological and Neurobehavioral Disorders in Mice. Curr. Drug Saf. 14: 199-208.

Holma, R., Korpela, R., Sairanen, U., Blom, M., Rautio, M., Poussa, T., et al. (2013). Colonic methane production modifies gastrointestinal toxicity associated with adjuvant 5 -fluorouracil chemotherapy for colorectal cancer. J. Clin. Gastroenterol. 47 : 45-51.

Huang, J.F., Zhao, Q., Dai, M.Y., Xiao, X.R., Zhang, T., Zhu, W.F., et al. (2020a). Gut microbiota protects from triptolide-induced hepatotoxicity: Key role of propionate and its downstream signalling events. Pharmacol. Res. 155 : 104752.

Huang, X., Fang, Q., Rao, T., Zhou, L., Zeng, X., Tan, Z., et al. (2020b). Leucovorin ameliorated methotrexate induced intestinal toxicity via modulation of the gut microbiota. Toxicol. Appl. Pharmacol.391 : 114900. 
Jarrott, B. (2017). Tacrine: In vivo veritas. Pharmacol. Res.116 : 29-31.

Jin, M.J., Kim, I.S., Kim, D.H., and Yoo, H.H. (2014). Effects of intestinal microbiota on the bioavailability of geniposide in rats. J. Agric. Food Chem.

Justino, P.F.C., Melo, L.F.M., Nogueira, A.F., Costa, J.V.G., Silva, L.M.N., Santos, C.M., et al. (2014). Treatment with Saccharomyces boulardii reduces the inflammation and dysfunction of the gastrointestinal tract in 5-fluorouracil-induced intestinal mucositis in mice. Br. J. Nutr.

Kang, M.J., Khanal, T., Kim, H.G., Lee, D.H., Yeo, H.K., Lee, Y.S., et al. (2012). Role of metabolism by human intestinal microflora in geniposideinduced toxicity in HepG2 cells. Arch. Pharm. Res. 35 : 733-738.

Khan, M.S., Ikram, M., Park, J.S., Park, T.J., and Kim, M.O. (2020). Gut Microbiota, Its Role in Induction of Alzheimer's Disease Pathology, and Possible Therapeutic Interventions: Special Focus on Anthocyanins. Cells.

Kim, J.K., Choi, M.S., Jeong, J.J., Lim, S.M., Kim, I.S., Yoo, H.H., et al. (2018). Effect of probiotics on pharmacokinetics of orally administered acetaminophen in mice. Drug Metab. Dispos. $46: 122-130$.

Kim, Y.C., and Lee, S.J. (1998). Temporal variation in hepatotoxicity and metabolism of acetaminophen in mice. Toxicology $128: 53-61$.

Klaassen, C.D., and Cui, J.Y. (2015). Review: Mechanisms of How the Intestinal Microbiota Alters the Effects of Drugs and Bile Acids. Drug Metab. Dispos.

Klein-Petersen, A.W., Köhler-Forsberg, O., and Benros, M.E. (2019). Infections, antibiotic treatment and the mircrobiome in relation to schizophrenia. Schizophr. Res.

Klein, R.M., Rigobello, C., Vidigal, C.B., Moura, K.F., Barbosa, D.S., Gerardin, D.C.C., et al. (2020). Gestational exposure to paracetamol in rats induces neurofunctional alterations in the progeny. Neurotoxicol. Teratol.

Koliada, A., Syzenko, G., Moseiko, V., Budovska, L., Puchkov, K., Perederiy, V., et al. (2017). Association between body mass index and Firmicutes/Bacteroidetes ratio in an adult Ukrainian population. BMC Microbiol. $17: 4-9$.

Krishna, G., and Muralidhara (2018). Oral supplements of combined fructo- and xylo-oligosaccharides during perinatal period significantly offsets acrylamide-induced oxidative impairments and neurotoxicity in rats. J. Physiol. Pharmacol. 69 : 801-814.

Lamba, V., Sangkuhl, K., Sanghavi, K., Fish, A., Altman, R.B., and Klein, T.E. (2014). PharmGKB summary: mycophenolic acid pathway. Pharmacogenet. Genomics.

Leclercq, S., Mian, F.M., Stanisz, A.M., Bindels, L.B., Cambier, E., Ben-Amram, H., et al. (2017). Low-dose penicillin in early life induces long-term changes in murine gut microbiota, brain cytokines and behavior. Nat. Commun.

Leung, C., Rivera, L., Furness, J.B., and Angus, P.W. (2016). The role of the gut microbiota in NAFLD. Nat. Rev. Gastroenterol. Hepatol.13 : 412-425.

Li, H., He, J., and Jia, W. (2016). The influence of gut microbiota on drug metabolism and toxicity. Expert Opin. Drug Metab. Toxicol.12 : 31-40.

Li, Y., Pan, H., Li, X., Jiang, N., Huang, L., Lu, Y., et al. (2019). Role of intestinal microbiota-mediated genipin dialdehyde intermediate formation in geniposide-induced hepatotoxicity in rats. Toxicol. Appl. Pharmacol. 377 :.

Liew, W.P.P., Mohd-Redzwan, S., and Than, L.T.L. (2019). Gut microbiota profiling of aflatoxin b1-induced rats treated with Lactobacillus casei shirota. Toxins (Basel). 
Lin, X., Zhao, J., Zhang, W., He, L., Wang, L., Chang, D., et al. (2020). Acute oral methylmercury exposure perturbs the gut microbiome and alters gut-brain axis related metabolites in rats. Ecotoxicol. Environ. Saf. $190: 110130$.

Liu, S., Marcelin, G., Blouet, C., Jeong, J.H., Jo, Y.H., Schwartz, G.J., et al. (2018). A gut-brain axis regulating glucose metabolism mediated by bile acids and competitive fibroblast growth factor actions at the hypothalamus. Mol. Metab.

Liu, Y., Li, Y., Xia, Y., Liu, K., Ren, L., and Ji, Y. (2020). The dysbiosis of gut microbiota caused by low-dose cadmium aggravate the injury of mice liver through increasing intestinal permeability. Microorganisms.

Madden, S., Woolf, T.F., Pool, W.F., and Park, B.K. (1993). An investigation into the formation of stable, protein-reactive and cytotoxic metabolites from tacrine in vitro. Studies with human and rat liver microsomes. Biochem. Pharmacol.

Mahmoodpoor, F., Rahbar Saadat, Y., Barzegari, A., Ardalan, M., and Zununi Vahed, S. V (2017). The impact of gut microbiota on kidney function and pathogenesis. Biomed. Pharmacother.

Malfatti, M.A., Kuhn, E.A., Murugesh, D.K., Mendez, M.E., Hum, N., Thissen, J.B., et al. (2020). Manipulation of the Gut Microbiome Alters Acetaminophen Biodisposition in Mice. Sci. Rep. 10 : 1-10.

Mancini, A., Campagna, F., Amodio, P., and Tuohy, K.M. (2018). Gut: Liver: brain axis: The microbial challenge in the hepatic encephalopathy. Food Funct. $9: 1373-1388$.

Neag, M.A., Catinean, A., Muntean, D.M., Pop, M.R., Bocsan, C.I., Botan, E.C., et al. (2020). Probiotic bacillus spores protect against acetaminophen induced acute liver injury in rats. Nutrients.

Nishida, A., Inoue, R., Inatomi, O., Bamba, S., Naito, Y., and Andoh, A. (2018). Gut microbiota in the pathogenesis of inflammatory bowel disease. Clin. J. Gastroenterol.

Park, S.M., Ki, S.H., Han, N.R., Cho, I.J., Ku, S.K., Kim, S.C., et al. (2015). Tacrine, an oral acetylcholinesterase inhibitor, induced hepatic oxidative damage, which was blocked by liquiritigenin through GSK3-beta inhibition. Biol. Pharm. Bull.

Peng, R., Ma, S.R., Fu, J., Han, P., Pan, L. Bin, Zhang, Z.W., et al. (2020). Transforming of triptolide into characteristic metabolites by the gut microbiota. Molecules 25 :.

Possamai, L.A., Mcphail, M.J., Khamri, W., Wu, B., Concas, D., Harrison, M., et al. (2015). The role of intestinal microbiota in murine models of acetaminophen-induced hepatotoxicity. Liver Int. 35 : 764-773.

Qin, J., Li, R., Raes, J., Arumugam, M., Burgdorf, K.S., Manichanh, C., et al. (2010). A human gut microbial gene catalogue established by metagenomic sequencing. Nature.

Quigley, E.M.M. (2017). Microbiota-Brain-Gut Axis and Neurodegenerative Diseases. Curr. Neurol. Neurosci. Rep.

Rasmussen, B.B., Nielsen, T.L., and Brøsen, K. (1998). Fluvoxamine is a potent inhibitor of the metabolism of caffeine in vitro. Pharmacol. Toxicol.

Relling, M. V., and Evans, W.E. (2015). Pharmacogenomics in the clinic. Nature.

Roberts, A.B., Wallace, B.D., Venkatesh, M.K., Mani, S., and Redinbo, M.R. (2013). Molecular insights into microbial $\beta$-glucuronidase inhibition to abrogate CPT-11 toxicity. Mol. Pharmacol.

Roberts, M.S., Magnusson, B.M., Burczynski, F.J., and Weiss, M. (2002). Enterohepatic circulation: Physiological, pharmacokinetic and clinical implications. Clin. Pharmacokinet.

Roman, P., Cardona, D., Sempere, L., and Carvajal, F. (2019). Microbiota and organophosphates. Neurotoxicology $75: 200-208$.

Roy, S., and Trinchieri, G. (2017). Microbiota: A key orchestrator of cancer therapy. Nat. Rev. Cancer. 
Saad, M.A., Rastanawi, A.A., and El-Yamany, M.F. (2018). Alogliptin abates memory injuries of hepatic encephalopathy induced by acute paracetamol intoxication via switching-off autophagy-related apoptosis. Life Sci. 215 : 11-21.

Scorza, C., Piccini, C., Martínez Busi, M., Abin Carriquiry, J.A., and Zunino, P. (2019). Alterations in the Gut Microbiota of Rats Chronically Exposed to Volatilized Cocaine and Its Active Adulterants Caffeine and Phenacetin. Neurotox. Res. 35 : 111-121.

Secombe, K.R., Coller, J.K., Gibson, R.J., Wardill, H.R., and Bowen, J.M. (2019). The bidirectional interaction of the gut microbiome and the innate immune system: Implications for chemotherapy-induced gastrointestinal toxicity. Int. J. Cancer.

Shen, T.C.D., Albenberg, L., Bittinger, K., Chehoud, C., Chen, Y.Y., Judge, C.A., et al. (2015). Engineering the gut microbiota to treat hyperammonemia. J. Clin. Invest. 125:2841-2850.

Sougiannis, A.T., VanderVeen, B.N., Enos, R.T., Velazquez, K.T., Bader, J.E., Carson, M., et al. (2019). Impact of 5 fluorouracil chemotherapy on gut inflammation, functional parameters, and gut microbiota. Brain. Behav. Immun.

Srivastav, S., Neupane, S., Bhurtel, S., Katila, N., Maharjan, S., Choi, H., et al. (2019). Probiotics mixture increases butyrate, and subsequently rescues the nigral dopaminergic neurons from MPTP and rotenoneinduced neurotoxicity. J. Nutr. Biochem. $69: 73-86$.

Stachlewitz, R.F., Arteel, G.E., Raleigh, J.A., Connor, H.D., Mason, R.P., and Thurman, R.G. (1997). Development and characterization of a new model of tacrine-induced hepatotoxicity: Role of the sympathetic nervous system and hypoxia- reoxygenation. J. Pharmacol. Exp. Ther.

Tang, D., Zeng, T., Wang, Y., Cui, H., Wu, J., Zou, B., et al. (2020). Dietary restriction increases protective gut bacteria to rescue lethal methotrexate-induced intestinal toxicity. Gut Microbes 00 : 1-21.

Taylor, M.R., Flannigan, K.L., Rahim, H., Mohamud, A., Lewis, I.A., Hirota, S.A., et al. (2019). Vancomycin relieves mycophenolate mofetil-induced gastrointestinal toxicity by eliminating gut bacterial -glucuronidase activity. Sci. Adv. $5: 1-10$.

Teichman, E.M., O'Riordan, K.J., Gahan, C.G.M., Dinan, T.G., and Cryan, J.F. (2020). When Rhythms Meet the Blues: Circadian Interactions with the Microbiota-Gut-Brain Axis. Cell Metab.

Thaiss, C.A., Levy, M., Korem, T., Dohnalová, L., Shapiro, H., Jaitin, D.A., et al. (2016). Microbiota Diurnal Rhythmicity Programs Host Transcriptome Oscillations. Cell 167 : 1495-1510.e12.

Tian, J., Zhu, J., Yi, Y., Li, C., Zhang, Y., Zhao, Y., et al. (2017). Dose-related liver injury of Geniposide associated with the alteration in bile acid synthesis and transportation. Sci. Rep. 7:1-11.

Tilg, H., Cani, P.D., and Mayer, E.A. (2016). Gut microbiome and liver diseases. Gut.

Vigo, M.B., Pérez, M.J., Fino, F. De, Gómez, G., Martínez, S.A., Bisagno, V., et al. (2019). Acute acetaminophen intoxication induces direct neurotoxicity in rats manifested as astrogliosis and decreased dopaminergic markers in brain areas associated with locomotor regulation. Biochem. Pharmacol.

Wacoo, A.P., Atukunda, P., Muhoozi, G., Braster, M., Wagner, M., Broek, T.J. van den, et al. (2020). Aflatoxins: Occurrence, exposure, and binding to lactobacillus species from the gut microbiota of rural ugandan children. Microorganisms.

Wallace, B.D., Roberts, A.B., Pollet, R.M., Ingle, J.D., Biernat, K.A., Pellock, S.J., et al. (2015). Structure and Inhibition of Microbiome $\beta$-Glucuronidases Essential to the Alleviation of Cancer Drug Toxicity. Chem. Biol. 22 : 1238-1249.

Wallace, B.D., Wang, H., Lane, K.T., Scott, J.E., Orans, J., Koo, J.S., et al. (2010). Alleviating Cancer Drug Toxicity by Inhibiting a Bacterial Enzyme. Science (80-. ). 831-836. 
Walsh, J., Griffin, B.T., Clarke, G., and Hyland, N.P. (2018). Drug-gut microbiota interactions: implications for neuropharmacology. Br. J. Pharmacol.

Wang, J., Feng, W., Zhang, S., Chen, L., Sheng, Y., Tang, F., et al. (2019a). Ameliorative effect of Atractylodes macrocephala essential oil combined with Panax ginseng total saponins on 5-fluorouracil induced diarrhea is associated with gut microbial modulation. J. Ethnopharmacol.

Wang, L., Yang, X., and Wu, H. (2019b). Juvenile Rats Show Altered Gut Microbiota After Exposure to Isoflurane as Neonates. Neurochem. Res.44: 776-786.

Wang, W., Zhou, H., and Liu, L. (2018). Side effects of methotrexate therapy for rheumatoid arthritis: A systematic review. Eur. J. Med. Chem. $158: 502-516$.

Wang, W.W., Zhang, Y., Huang, X.B., You, N., Zheng, L., and Li, J. (2017). Fecal microbiota transplantation prevents hepatic encephalopathy in rats with carbon tetrachloride-induced acute hepatic dysfunction. World J. Gastroenterol.

Wang, Y., Sun, L., Chen, S., Guo, S., Yue, T., Hou, Q., et al. (2019c). The administration of Escherichia coli Nissle 1917 ameliorates irinotecan-induced intestinal barrier dysfunction and gut microbial dysbiosis in mice. Life Sci.

Wardill, H.R., and Tissing, W.J.E. (2017). Determining risk of severe gastrointestinal toxicity based on pretreatment gut microbial community in patients receiving cancer treatment: A new predictive strategy in the quest for personalized cancer medicine. Curr. Opin. Support. Palliat. Care.

Weir, V. (2020). Nonpharmacologic Management of Hepatic Encephalopathy: An Update. Clin. Liver Dis.

Wiest, R., Albillos, A., Trauner, M., Bajaj, J.S., and Jalan, R. (2017). Targeting the gut-liver axis in liver disease. J. Hepatol.

Wijdicks, E.F.M. (2016). Hepatic Encephalopathy. N. Engl. J. Med.

Wu, H., Rao, Q., Ma, G.C., Yu, X.H., Zhang, C.E., and Ma, Z.J. (2020). Effect of triptolide on dextran sodium sulfate-induced ulcerative colitis and gut microbiota in mice. Front. Pharmacol. 10 : 1-13.

Xi, C., Peng, S., Wu, Z., Zhou, Q., and Zhou, J. (2017). Toxicity of triptolide and the molecular mechanisms involved. Biomed. Pharmacother.90 : 531-541.

Xue, H., Xie, W., Jiang, Z., Wang, M., Wang, J., Zhao, H., et al. (2016). 3,4-Dihydroxyphenylacetic acid, a microbiota-derived metabolite of quercetin, attenuates acetaminophen (APAP)-induced liver injury through activation of Nrf-2. Xenobiotica $46: 931-939$.

Yamano, T., Noda, T., Shimizu, M., Ohmorz, M., Morita, S., and Yamada, A. (1990). Hepatotoxicity of Geniposide in Rats. Food Chem Toxicol28 : 515-519.

Yang, J., Sun, L., Wang, L., Hassan, H.M., Wang, X., Hylemon, P.B., et al. (2017). Activation of sirt1/FXR signaling pathway attenuates triptolide-induced hepatotoxicity in rats. Front. Pharmacol. $8: 1-11$.

Yang, S., Kuang, G., Jiang, R., Wu, S., Zeng, T., Wang, Y., et al. (2019). Geniposide protected hepatocytes from acetaminophen hepatotoxicity by down-regulating CYP $2 \mathrm{E} 1$ expression and inhibiting TLR 4/NF- $x \mathrm{~B}$ signaling pathway. Int. Immunopharmacol. 74: 105625.

Yang, T., Richards, E.M., Pepine, C.J., and Raizada, M.K. (2018a). The gut microbiota and the brain-gutkidney axis in hypertension and chronic kidney disease. Nat. Rev. Nephrol.

Yang, W., Wei, B., and Yan, R. (2018b). Amoxapine Demonstrates Incomplete Inhibition of $\beta$-Glucuronidase Activity from Human Gut Microbiota. SLAS Discov. 23 : 76-83.

Yip, L.Y., Aw, C.C., Lee, S.H., Hong, Y.S., Ku, H.C., Xu, W.H., et al. (2018). The liver-gut microbiota axis modulates hepatotoxicity of tacrine in the rat. Hepatology 67 : 282-295. 
Zeng, S.L., Li, S.Z., Xiao, P.T., Cai, Y.Y., Chu, C., Chen, B.Z., et al. (2020). Citrus polymethoxyflavones attenuate metabolic syndrome by regulating gut microbiome and amino acid metabolism. Sci. Adv. 6 : $1-14$.

Zhang, J., Zhang, J., and Wang, R. (2018). Gut microbiota modulates drug pharmacokinetics. Drug Metab. Rev.

Zhang, L., Ye, Y., An, Y., Tian, Y., Wang, Y., and Tang, H. (2011). Systems responses of rats to aflatoxin B1 exposure revealed with metabonomic changes in multiple biological matrices. J. Proteome Res.

Zhang, S., Jin, Y., Zeng, Z., Liu, Z., and Fu, Z. (2015). Subchronic Exposure of Mice to Cadmium Perturbs Their Hepatic Energy Metabolism and Gut Microbiome. Chem. Res. Toxicol.

Zhao, H., Jiang, Z., Chang, X., Xue, H., Yahefu, W., and Zhang, X. (2018). 4-hydroxyphenylacetic acid prevents acute APAP-induced liver injury by increasing phase II and antioxidant enzymes in mice. Front. Pharmacol. 9:1-10.

Zhou, B., Xia, X., Wang, P., Chen, S., Yu, C., Huang, R., et al. (2018a). Induction and Amelioration of Methotrexate-Induced Gastrointestinal Toxicity are Related to Immune Response and Gut Microbiota. EBioMedicine 33 : 122-133.

Zhou, J., Tang, L., Wang, J., and Wang, J.S. (2018b). Aflatoxin B1 disrupts gut-microbial metabolisms of short-chain fatty acids, long-chain fatty acids, and bile acids in male F344 rats. Toxicol. Sci.

Zhou, J., Tang, L., and Wang, J.S. (2019a). Assessment of the adverse impacts of aflatoxin B1 on gutmicrobiota dependent metabolism in F344 rats. Chemosphere.

Zhou, Y.X., Zhang, R.Q., Rahman, K., Cao, Z.X., Zhang, H., and Peng, C. (2019b). Diverse Pharmacological Activities and Potential Medicinal Benefits of Geniposide. Evidence-Based Complement. Altern. Med.2019 $\therefore$

Zimmermann, M., Zimmermann-Kogadeeva, M., Wegmann, R., and Goodman, A.L. (2019). Separating host and microbiome contributions to drug pharmacokinetics and toxicity. Science (80-. ). 

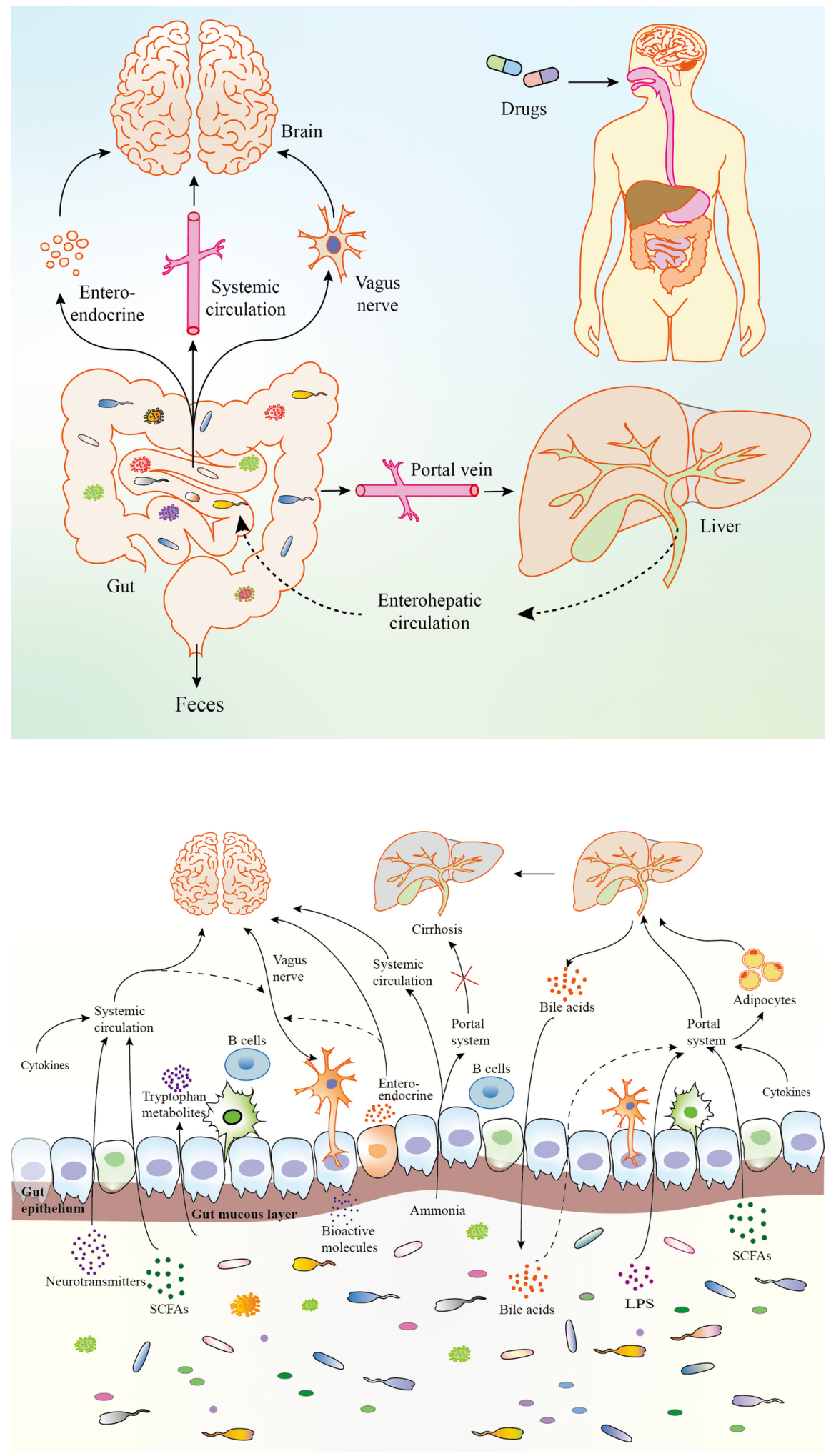

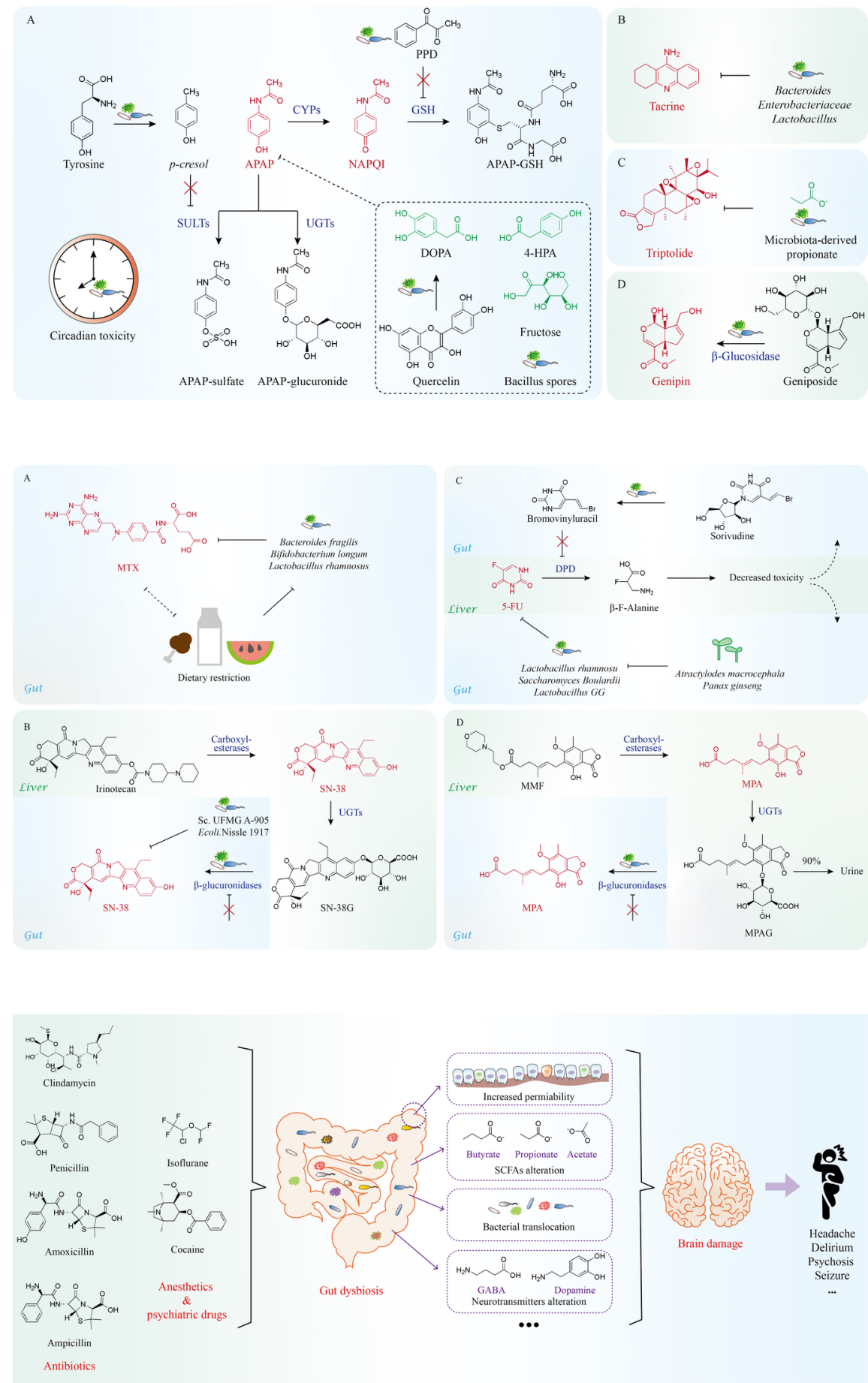

\section{Hosted file}

table 1.docx available at https://authorea.com/users/330867/articles/457672-connecting-thedots-targeting-gut-microbiota-in-drug-toxicity 